MITH-96/2

\title{
A possible scaling region of chiral fermions on a lattice
}

\author{
She-Sheng Xue ${ }^{(a)}$ \\ INFN - Section of Milan, Via Celoria 16, Milan, Italy
}

\begin{abstract}
We present the details of analyzing an $S U_{L}(2) \otimes U_{R}(1)$ chiral theory with multifermion couplings on a lattice. An existence of a possible scaling region in the phase space of multifermion couplings for defining the continuum limit of chiral fermions is advocated. In this scaling region, no spontaneous symmetry breaking occurs; the "spectator" fermion $\psi_{R}(x)$ is a free mode and decoupled; doublers are decoupled as massive Dirac fermions consistently with the $S U_{L}(2) \otimes$ $U_{R}(1)$ chiral symmetry, whereas the normal mode of $\psi_{L}^{i}(x)$ is plausibly speculated to be chiral in the continuum limit. This is not in agreement with the general belief of the definite failure of theories so constructed.
\end{abstract}

March, 1996

PACS 11.15Ha, 11.30.Rd, 11.30.Qc

a) E-mail address: xue@milano.infn.it 


\section{Introduction}

Since the "no-go" theorem [1] of Nielsen and Ninomiya was demonstrated in 1981 the problem of chiral fermion "doubling" and "vector-like" phenomenon on a lattice still exists if one insists on preserving chiral symmetry. One of the ideas to get around this "no-go" theorem was proposed by Eichten and Preskill (EP) [2] ten years ago. The crucial points of this idea can be briefly described as follows. Multifermion couplings are introduced such that, in the phase space of strongcouplings, Weyl states composing three elementary Weyl fermions (three-fermion states) are bound. Then, these three-fermion states pair up with elementary Weyl fermions to be Dirac fermions. Such Dirac fermions can be massive without violating chiral symmetries due to the appropriate quantum numbers and chirality carried by these three-fermion states. The binding thresholds of such threefermion states depend on elementary Weyl modes residing in different regions of the Brillouin zone. If one assumes that the spontaneous symmetry breaking of the Nambu-Jona Lasinio (NJL) type does not occur and such binding thresholds separate the weak coupling symmetric phase from the strong-coupling symmetric phase, there are two possibilities to realize the continuum limit of chiral fermions in phase space. One is of crossing over the binding threshold the three-fermion state of chiral fermions. Another is of a wedge between two thresholds, where the three-fermion state of chiral fermions has not been formed, provided all doublers sitting in various edges of the Brillouin zone have been bound to be massive Dirac fermions and decouple.

To visualize this idea, EP proposed a model [2] of multifermion couplings with $S U(5)$ and $S O(10)$ chiral symmetries and suggested the possible regions in phase space to define the continuum limit of chiral fermions. However, the same model of multifermion couplings with $S O(10)$ chiral symmetry was studied in ref. [3], it was pointed out that such models of multifermion couplings fail to give chiral fermions in the continuum limit. The reasons 1 are that an NJL spontaneous symmetry breaking phase separating the strong-coupling symmetric phase from the weak-coupling symmetric phase, the right-handed Weyl states do not completely disassociate from the left-handed chiral fermions and the phase structure of such a model of multifermion couplings is similar to that of the Smit-Swift (Wilson-Yukawa) model [4], which has been very carefully studied and shown to fail. It is a general belief [5] that the constructions [2, 6, 15] of chiral fermions on the lattice with multifermion couplings must fail to give chiral fermions on the basis of a general opinion that multifermion couplings and Yukawa couplings should be in the same universality class. In fact, this opinion is indeed correct if one considers multifermion couplings and Yukawa couplings only for continuous field theory in the sense that two theories have the same spectrum and relevant operators at the ultra-violate fix point [7]. However, it is hard to prove

\footnotetext{
${ }^{1}$ I thank M.F.L. Golterman and D.N. Petcher for discussions on this subject.
} 
this opinion by showing a one-to-one correspondence between two phase spaces of multifermion couplings and Yukawa couplings on a lattice, where they have the exactly same spectra and relevant operators not only for chiral fermions but also for doublers. Even for Yukawa couplings, models with different symmetries could be in different universality classes [8]. Generally speaking, multifermion couplings possess more symmetries than Yukawa couplings in a lattice theory. All symmetries of the Standard Model can possibly be preserved by multifermion couplings, but not Wilson-Yukawa couplings on a lattice.

We should not be surprised that a particular model of multifermion couplings does not work. This does not means that EP's idea is definitely wrong unless there is another generalized "no-go" theorem on interacting theories [9] for a whole range of coupling strength; we will come back to this point in section 6. Actually, Nielsen and Ninomiya gave an interesting comment on EP's idea based on their intuition of anomalies [10]. As a matter of fact, the phase space of multifermion coupling models of the EP type [2, 3] has not been completely explored. To conclude, we believe that further considerations of constructing chiral fermions on the lattice with multifermion couplings and careful studies of the spectrum in each phase of theories so constructed are necessary.

Note that exploring a possible scaling region of the continuum limit of lattice (non-gauged) chiral fermions in the phase space of multifermion couplings is the main goal of this paper 2 . This is tightly related to many problems in particle physics 11. We are not pretending to solve all problems of lattice chiral gauge theories in this paper. If chiral fermions are gauged, the important questions concerning correct and consistent features of gauge bosons and the coupling between gauge bosons and chiral fermions are open, but beyond the scope of this paper. Other important questions [12] concerning about gauge anomalies and anomalous global currents (instanton effects and non-conservation of fermion currents), which EP [2] suggested to obtain by explicitly breaking the global symmetries associating to these currents, will be studied in separate papers.

In section 2, we present a model of chiral fermions with multifermion couplings on the lattice and discuss the $\psi_{R}(x)$ shift-symmetry and its related Ward identity. The analyses of the weak-coupling phase and the strong-coupling phase are given in sections 3 and 4 . The thresholds and wedges that EP expected are qualitatively determined and discussed in section 5. An existence of a possible scaling region for the continuum limit of lattice chiral fermions is advocated and discussed in section 6 . In the last section, we make some remarks on problems and possible resolutions if this model is chirally gauged.

\footnotetext{
${ }^{2}$ The short version of this paper is published in Phys. Lett. B381 (1996) 277.
} 


\section{Formulation and the $\psi_{R}$ shift-symmetry}

Let us consider the following action of chiral fermions with the $S U_{L}(2) \otimes U_{R}(1)$ global chiral symmetry on the lattice.

$$
\begin{aligned}
S & =S_{f}+S_{1}+S_{2} \\
S_{f} & =\frac{1}{2 a} \sum_{x} \sum_{\mu}\left(\bar{\psi}_{L}^{i}(x) \gamma_{\mu} D_{i j}^{\mu} \psi_{L}^{j}(x)+\bar{\psi}_{R}(x) \gamma_{\mu} \partial^{\mu} \psi_{R}(x)\right) \\
S_{1} & =g_{1} \sum_{x} \bar{\psi}_{L}^{i}(x) \cdot \psi_{R}(x) \bar{\psi}_{R}(x) \cdot \psi_{L}^{i}(x) \\
S_{2} & =g_{2} \sum_{x} \bar{\psi}_{L}^{i}(x) \cdot\left[\Delta \psi_{R}(x)\right]\left[\Delta \bar{\psi}_{R}(x)\right] \cdot \psi_{L}^{i}(x) .
\end{aligned}
$$

In eq. (1),$S_{f}$ is the naive lattice action of chiral fermions, $a$ is the lattice spacing and the $S U_{L}(2)$ chiral symmetry is actually local and can easily be gauged

$$
\sum_{\mu} \gamma_{\mu} D^{\mu}=\sum_{\mu}\left(U_{\mu}(x) \delta_{x, x+\mu}-U_{\mu}^{\dagger}(x) \delta_{x, x-\mu}\right), \quad U_{\mu}(x) \in S U_{L}(2),
$$

but we will impose $U_{\mu}(x)=1$ so that the $S U_{L}(2)$ is a global symmetry. $S_{1}$ and $S_{2}$ are two external multifermion couplings, where

$$
\begin{aligned}
\Delta \psi_{R}(x) & \equiv \sum_{\mu}\left[\psi_{R}(x+\mu)+\psi_{R}(x-\mu)-2 \psi_{R}(x)\right] \\
\Delta \bar{\psi}_{R}(x) & \equiv \sum_{\mu}\left[\bar{\psi}_{R}(x+\mu)+\bar{\psi}_{R}(x-\mu)-2 \bar{\psi}_{R}(x)\right] .
\end{aligned}
$$

In the action (11), $\psi_{L}^{i}(i=1,2)$ is an $S U_{L}(2)$ gauged doublet, $\psi_{R}$ is an $S U_{L}(2)$ singlet and both are two-component Weyl fermions. $\psi_{R}$ is treated as a "spectator" fermion. $\psi_{L}^{i}$ and $\psi_{R}$ fields are dimensionful $\left[a^{-\frac{1}{2}}\right]$. The first multifermion coupling $S_{1}$ in eq. (1) is a dimension-6 operator relevant both for doublers $p=\tilde{p}+\pi_{A}$ and for normal modes $p=\tilde{p}$ of $\psi_{L}^{i}$ and $\psi_{R}$ fields. Note that all momenta are scaled to be dimensionless, the physical momentum $(\tilde{p})$ of normal modes and the momentum $p=\tilde{p}+\pi_{A}$ of doublers are

$$
\tilde{p} \simeq 0, \quad p=\tilde{p}+\pi_{A},
$$

where $\pi_{A}$ runs over fifteen lattice momenta $\pi_{A} \neq 0$. The second multifermion coupling $S_{2}$ in eq. (1) is a dimension-10 operator relevant only for doublers, but irrelevant for normal modes of $\psi_{L}^{i}$ and $\psi_{R}$. The multifermion couplings $g_{1}$ and $g_{2}$ have dimension $\left[a^{-2}\right]$. The $S_{1}$ is similar to the mass term in lattice QCD and the second term is similar to the Wilson term. They are quadrilinear in order to preserve chiral gauge symmetries.

The action (1) has an exact local $S U(2)$ chiral gauge symmetry, which is the symmetry that the continuum theory (the target theory) possesses. The global 
flavour symmetry $U_{L}(1) \otimes U_{R}(1)$ is not explicitly broken in eq. (11). When $g_{1}=0$, the action (11) possesses a $\psi_{R}$ shift-symmetry [13], i.e., the action is invariant under the transformation:

$$
\bar{\psi}_{R}(x) \rightarrow \bar{\psi}_{R}(x)+\bar{\epsilon}, \quad \psi_{R}(x) \rightarrow \psi_{R}(x)+\epsilon
$$

where $\epsilon$ is independent of space-time.

To derive the Ward identity associated with this $\psi_{R}(x)$-shift-symmetry (5), we consider the generating functional $W(\eta, J)$ and partition functional $Z(\eta, J)$ of the theory,

$$
\begin{aligned}
W(\eta, J) & =-\ln Z(\eta, J), \\
Z(\eta, J) & =\int_{\phi} \exp \left(-S+\int_{x}\left(\bar{\psi}_{L}^{i} \eta_{L}^{i}+\bar{\eta}_{L}^{i} \psi_{L}^{i}+\bar{\psi}_{R} \eta_{R}+\bar{\eta}_{R} \psi_{R}+A_{\mu} J^{\mu}\right)\right), \\
\int_{\phi} & =\int\left[d \psi_{L}^{i} d \psi_{R} d A_{\mu}\right],
\end{aligned}
$$

where $A_{\mu}(x)$ refers to the $S U_{L}(2)$ gauge field (2) defined on the lattice. Then we define the generating functional of one-particle irreducible vertices (the effective action $\left.\Gamma\left(\psi_{L}^{\prime i}, \psi_{R}^{\prime}, A_{\mu}^{\prime}\right)\right)$ as the Legendre transform of $W(\eta, J)$,

$$
\Gamma\left(\psi_{L}^{\prime i}, \psi_{R}^{\prime}, A_{\mu}^{\prime}\right)=W(\eta, J)-\int_{x}\left(\bar{\psi}_{L}^{\prime i} \eta_{L}^{i}+\bar{\eta}_{L}^{i} \psi_{L}^{\prime i}+\bar{\psi}_{R}^{\prime} \eta_{R}+\bar{\eta}_{R} \psi_{R}^{\prime}+A_{\mu}^{\prime} J^{\mu}\right)
$$

with the relations

$$
\begin{array}{ll}
A_{\mu}^{\prime}(x)=\left\langle A_{\mu}(x)\right\rangle=-\frac{\delta W}{\delta J_{\mu}(x)}, & \\
\psi_{L}^{\prime i}(x)=\left\langle\psi_{L}^{i}(x)\right\rangle=-\frac{\delta W}{\delta \bar{\eta}_{L}^{i}(x)}, & \bar{\psi}_{L}^{\prime i}(x)=\left\langle\bar{\psi}_{L}^{i}(x)\right\rangle=\frac{\delta W}{\delta \eta_{L}^{i}(x)} \\
\psi_{R}^{\prime}(x)=\left\langle\psi_{R}(x)\right\rangle=-\frac{\delta W}{\delta \bar{\eta}_{R}(x)}, & \bar{\psi}_{R}^{\prime}(x)=\left\langle\bar{\psi}_{R}(x)\right\rangle=\frac{\delta W}{\delta \eta_{R}(x)}
\end{array}
$$

in which the fermionic derivatives are left-derivatives, and

$$
\begin{array}{rlrl}
J_{\mu}(x) & =-\frac{\delta \Gamma}{\delta A_{\mu}^{\prime}(x)}, & & \\
\eta_{L}^{i}(x) & =-\frac{\delta \Gamma}{\delta \bar{\psi}_{L}^{\prime i}(x)}, & \bar{\eta}_{L}^{i}(x)=\frac{\delta \Gamma}{\delta \psi_{L}^{\prime i}(x)} \\
\eta_{R}(x)=-\frac{\delta \Gamma}{\delta \bar{\psi}_{R}^{\prime}(x)}, & \bar{\eta}_{R}(x)=\frac{\delta \Gamma}{\delta \psi_{R}^{\prime}(x)} .
\end{array}
$$

In eqs. (9, 10], the $\langle\cdots\rangle$ indicates

$$
\langle\cdots\rangle=\frac{1}{Z} \int_{\phi}(\cdots) \exp \left(-S+\int_{x}\left(\bar{\psi}_{L}^{i} \eta_{L}^{i}+\bar{\eta}_{L}^{i} \psi_{L}^{i}+\bar{\psi}_{R} \eta_{R}+\bar{\eta}_{R} \psi_{R}+A_{\mu} J^{\mu}\right)\right),
$$


which is an expectation value with respect to the partition functional $Z(\eta, J)$.

Making the parameter $\epsilon$ to be space-time dependent, and varying the generating functional (6) according to the transformation rules (5) for arbitrary $\epsilon(x) \neq 0$, we arrive at

$\bar{\epsilon}(x)\left\langle\frac{1}{2 a} \gamma_{\mu} \partial^{\mu} \psi_{R}(x)+g_{1} \bar{\psi}_{L}^{i}(x) \cdot \psi_{R}(x) \psi_{L}^{i}(x)+g_{2} \Delta\left(\bar{\psi}_{L}^{i}(x) \cdot \Delta \psi_{R}(x) \psi_{L}^{i}(x)\right)+\eta_{R}(x)\right\rangle=0$.

Together with (12), the Ward identity in terms of the primed fields corresponding to the $\psi_{R}$ shift-symmetry of the action (臬) is given as

$\frac{1}{2 a} \gamma_{\mu} \partial^{\mu} \psi_{R}^{\prime}(x)+g_{1}\left\langle\bar{\psi}_{L}^{i}(x) \cdot \psi_{R}(x) \psi_{L}^{i}(x)\right\rangle+g_{2}\left\langle\Delta\left(\bar{\psi}_{L}^{i}(x) \cdot \Delta \psi_{R}(x) \psi_{L}^{i}(x)\right)\right\rangle-\frac{\delta \Gamma}{\delta \bar{\psi}_{R}^{\prime}(x)}=0$.

Based on this Ward identity, one can get all one-particle irreducible vertices containing at least one external $\psi_{R}$.

Taking the functional derivative of eq. (15) with respect to $\psi_{R}^{\prime}(0)$ and then putting external sources $\eta=0$ and $J=0$, we derive (see appendix I):

$$
\begin{aligned}
& {\left[\frac{1}{2 a}\left(\gamma_{\mu}\right)^{\beta \alpha} \partial^{\mu} P_{R}-g_{1}\left\langle\bar{\psi}_{L}^{i \alpha}(x) \psi_{L}^{i \beta}(x)\right\rangle_{\circ}-g_{2} \Delta\left(\left\langle\bar{\psi}_{L}^{i \alpha}(x) \psi_{L}^{i \beta}(x)\right\rangle_{\circ} \Delta\right)\right] \delta(x)} \\
& -\frac{\delta^{2} \Gamma}{\delta \psi_{R}^{\prime \alpha}(0) \delta \bar{\psi}_{R}^{\prime \beta}(x)}=0
\end{aligned}
$$

where $\langle\cdots\rangle_{\circ}$ is the expectation value (13) with vanishing external sources $\eta$ and $J_{\mu}$. In eqs. (126,127) of appendix I, we show

$$
\left\langle\bar{\psi}_{L}^{i \alpha}(x) \psi_{L}^{i \beta}(x)\right\rangle_{\circ}=0, \quad \Delta\left\langle\bar{\psi}_{L}^{i \alpha}(x) \psi_{L}^{i \beta}(x)\right\rangle_{\circ}=0 .
$$

Thus, the two-point function in eq. (16) is given as,

$$
\int_{x} e^{-i p x} \frac{\delta^{(2)} \Gamma}{\delta \psi_{R}^{\prime}(x) \delta \bar{\psi}_{R}^{\prime}(0)}=\frac{i}{a} \gamma_{\mu} \sin \left(p^{\mu} a\right),
$$

which shows that $\psi_{R}$ does not receive wave-function renormalization.

\section{The weak-coupling region}

Our goal is to seek a possible regime, where an undoubled $S U_{L}(2)$ chiral gauged fermion content is exhibited in the continuum limit in the phase space $\left(g_{1}, g_{2}, g\right)$, where " $g$ " is the gauge coupling, regarded to be a truly small perturbation $g \rightarrow 0$ at the scale of the continuum limit we consider. Thus, we impose $g=0$ and $U_{\mu}(x)=1$ in eq. (2). In the weak-coupling limit, $g_{1} \ll 1$ and $g_{2} \ll 1$ (indicated 1 in Fig. 1), the action (1) defines an $S U_{L}(2) \otimes U_{R}(1)$ chiral continuum theory with 
a doubled and weakly interacting fermion spectrum that is not the continuum theory we seek.

Let us consider the phase of a spontaneous symmetry breaking in the weakcoupling $g_{1}, g_{2}$ limit. Based on the analysis of large- $N_{f}\left(N_{f}\right.$ is an extra fermion index, e.g., color, $N_{c}$ ) weak-coupling expansion, we show that the multifermion couplings in the action (11) undergo Nambu-Jona Lasinio (NJL) spontaneous chiral-symmetry breaking [14. In this symmetry breaking phase indicated 2 in Fig. 1, the $S U_{L}(2) \otimes U_{R}(1)$ chiral symmetry is violated by

$$
\frac{1}{2} \Sigma^{i}(p)=g_{1} \int d^{4} x e^{-i p x}\left\langle\bar{\psi}_{L}^{i}(0) \cdot \psi_{R}(x)\right\rangle_{\circ} \neq 0 .
$$

Assuming that the symmetry breaking takes place in the direction 1 in the 2dimensional space of the $S U_{L}(2)$ chiral symmetry $\left(\Sigma^{1}(p) \neq 0, \Sigma^{2}(p)=0\right)$, one finds the following fermion spectrum that contains a doubled Weyl fermion $\psi_{L}^{2}(x)$ and an undoubled Dirac fermion made up of the Weyl fermions $\psi_{L}^{1}(x)$ and $\psi_{R}(x)$. The propagators of these fermions can be written as,

$$
\begin{aligned}
S_{b 1}^{-1}(p) & =\frac{i}{a} \sum_{\mu} \gamma_{\mu} \sin p^{\mu} Z_{2}(p) P_{L}+\frac{i}{a} \sum_{\mu} \gamma_{\mu} \sin p^{\mu} P_{R}+\Sigma^{1}(p) \\
S_{b 2}^{-1}(p) & =\frac{i}{a} \sum_{\mu} \gamma_{\mu} \sin p^{\mu} Z_{2}(p) P_{L} .
\end{aligned}
$$

The $S U_{L}(2) \otimes U_{R}(1)$ chiral symmetry is realized to be $U_{L}(1) \otimes U(1)$ with three Goldstone modes and a massive Higgs mode that are not presented in this report [15]. The fermion self-energy function $\Sigma^{1}(p)$ (19) for $i=1$ is given by

$$
\frac{1}{2} \Sigma^{1}(p)=\int d^{4} x e^{-i p x} \frac{\delta^{(2)} \Gamma}{\delta \psi_{L}^{\prime 1}(x) \delta \bar{\psi}_{R}^{\prime}(0)}
$$

The wave-function renormalization $Z_{2}(p)$ of $\psi_{L}^{i}(x)$ field is defined as

$$
\frac{i}{a} \gamma_{\mu} \sin p^{\mu} \delta_{i j} Z_{2}(p)=\int d^{4} x e^{-i p x} \frac{\delta^{(2)} \Gamma}{\delta \psi_{L}^{\prime i}(x) \delta \bar{\psi}_{L}^{\prime j}(0)}
$$

The wave-function renormalization of $\psi_{R}(x)$ field is fixed by eq. (18).

Based on the Ward identity (15) of the $\psi_{R}$ shift-symmetry, one can obtain an identity for the self-energy function $\Sigma^{i}(p)(19)$. Performing a functional derivative of eq. (15) with respect to $\psi_{L}^{\prime i}(0)$ and then putting external sources $\eta=0$ and $J=0$, and we obtain (see eqs. (128, 129) in appendix I)

$$
g_{1}\left\langle\bar{\psi}_{L}^{i}(x) \cdot \psi_{R}(x)\right\rangle_{\circ} \delta(x)+g_{2}\left\langle\Delta\left(\bar{\psi}_{L}^{i}(x) \cdot \Delta \psi_{R}(x) \delta(x)\right)\right\rangle_{\circ}-\frac{\delta^{2} \Gamma}{\delta \psi_{L}^{\prime i}(0) \delta \bar{\psi}_{R}^{\prime}(x)}=0
$$

Transforming into momentum space, we obtain

$$
\frac{1}{2} \Sigma^{i}(p)=g_{1}\left\langle\bar{\psi}_{L}^{i}(0) \cdot \psi_{R}(0)\right\rangle_{\circ}+2 g_{2} w(p)\left\langle\bar{\psi}_{L}^{i}(0) \cdot \Delta \psi_{R}(0)\right\rangle_{\circ}
$$


where the well-known Wilson factor [16] is

$$
\begin{aligned}
w(p) & =\sum_{\mu}\left(1-\cos \left(p_{\mu} a\right)\right), \\
2 w(p) & =\Delta(p)=\int d^{4} x e^{-i p x} \Delta(x) .
\end{aligned}
$$

As for the four-fermion interaction vertex, analogously, one takes functional derivatives of the Ward identity (15) with respect to $\bar{\psi}_{L}^{\prime i}(0), \psi_{L}^{\prime i}(y)$ and $\psi_{R}^{\prime}(z)$ and obtains

$$
\int_{x y z} e^{-i y q-i x p-i z p^{\prime}} \frac{\delta^{(4)} \Gamma}{\delta \psi_{L}^{\prime i}(0) \delta \bar{\psi}_{L}^{\prime i}(y) \delta \psi_{R}^{\prime}(z) \delta \bar{\psi}_{R}^{\prime}(x)}=g_{1}+4 g_{2} w\left(p+\frac{q}{2}\right) w\left(p^{\prime}+\frac{q}{2}\right),
$$

where $p+\frac{q}{2}$ and $p^{\prime}+\frac{q}{2}$ are the momenta of $\psi_{R}(x)$ field; $p-\frac{q}{2}$ and $p^{\prime}-\frac{q}{2}$ are the momenta of $\psi_{L}^{i}(x)$ field ( $q$ is the momentum transfer as shown in Fig. 2.). These two identities eqs (25,27) show us two consequences of the $\psi_{R}$ shift-symmetry when $g_{1}=0$ : (i) the normal modes of $\psi_{L}^{i}$ and $\psi_{R}$ are massless

$$
\Sigma^{i}(0)=0, \quad(O(a))
$$

(ii) the normal modes of $\psi_{R}(x)$ and $\psi_{L}^{i}(x)$ are free $\left(O\left(a^{2}\right)\right)$ from the four-fermion interaction, only the doublers of $\psi_{R}(x)$ and $\psi_{L}^{i}(x)$ have a non-vanishing fourfermion interacting. We will come back to these two points in sections 5 and 6.

Owing to the four-fermion interaction vertex (27), the fermion self-energy function $\Sigma^{1}(p)$ in eqs. (19) and (22) obeys the NJL gap-equation in the large- $N_{f}$ weak-coupling expansion $\left(N_{f} \rightarrow \infty\right)$ as shown in Fig. 3,

$$
\Sigma^{1}(p)=4 \int_{q} \frac{\Sigma^{1}(q)}{\operatorname{den}(q)}\left(\tilde{g}_{1}+4 \tilde{g}_{2} w(p) w(q)\right)
$$

where

$$
\begin{aligned}
\int_{q} & \equiv \int_{-\pi}^{\pi} \frac{d^{4} q}{(2 \pi)^{4}} \\
\operatorname{den}(q) & \equiv \sum_{\rho} \sin ^{2} q_{\rho}+\left(\Sigma^{1}(q) a\right)^{2} \\
\tilde{g}_{1} & \equiv g_{1} N_{f} a^{2}, \quad \tilde{g}_{2} \equiv g_{2} N_{f} a^{2} .
\end{aligned}
$$

We adopt the parametrization [3]

$$
\Sigma^{1}(p)=\Sigma^{1}(0)+\tilde{g}_{2} v^{1} w(p), \quad \Sigma^{1}(0)=\rho v^{1},
$$

where $\rho$ depends only on couplings $\tilde{g}_{1}, \tilde{g}_{2}$, and $v^{1}$ plays a role as the v.e.v. violating $S U_{L}(2) \otimes U_{R}(1)$ chiral symmetry. We can solve the gap-equation (29) by using this parametrization. For $v^{1}=O\left(\frac{1}{a}\right)$, one obtains (see appendix II)

$$
\rho=\frac{\tilde{g}_{1} \tilde{g}_{2} I_{1}}{1-\tilde{g}_{1} I_{\circ}} ; \quad \rho=\frac{1-4 \tilde{g}_{2} I_{2}}{4 I_{1}}
$$


where the functions $I_{n}\left(v^{1}\right),(n=0,1,2)$, are defined as

$$
I_{n}\left(v^{1}\right)=4 \int_{q} \frac{w^{n}(q)}{\sum_{\rho} \sin ^{2} q_{\rho}+\left(\Sigma^{1}(q) a\right)^{2}} .
$$

eq. (31) leads to a crucial result:

$$
\tilde{g}_{1}=0, \quad \rho=0 \quad \text { and } \quad \Sigma^{1}(0)=0,
$$

this is due to eq. (25), resulting from the Ward identity (15). This means that on the line $g_{1}=0$, the normal modes $(p=\tilde{p} \simeq 0)$ of $\psi_{L}^{1}$ and $\psi_{R}$ are massless and their 15 doublers $\left(p=\tilde{p}+\pi_{A}\right)$ acquire chiral-variant masses

$$
\Sigma^{1}(p)=\tilde{g}_{2} v^{1} w(p)
$$

through the multifermion coupling $g_{2}$ only. In this case $\left(g_{1}=0\right)$, the gap-equation is then given by eq. (31) for $\rho=0$,

$$
1-4 \tilde{g}_{2} I_{2}\left(v^{1}\right)=0, \quad \text { i.e. } \quad 1=16 \tilde{g}_{2} \int_{q} \frac{w^{2}(q)}{\sum_{\rho} \sin ^{2} q_{\rho}+\left(\tilde{g}_{2} v^{1} w(q) a\right)^{2}} .
$$

The Wilson factor $w^{2}(q)$ contained in integral (35) indicates that only doublers contribute to the NJL gap-equation.

As $v^{1} \rightarrow 0$, eq. (31) gives a critical line $\tilde{g}_{1}^{c}\left(\tilde{g}_{2}^{c}\right)$ :

$$
\tilde{g}_{1}^{c}=\frac{1-4 \tilde{g}_{2}^{c} I_{2}(0)}{4 \tilde{g}_{2}^{c} I_{1}^{2}(0)+I_{\circ}(0)-4 \tilde{g}_{2}^{c} I_{\circ}(0) I_{2}(0)},
$$

characterizing the NJL spontaneous symmetry breaking. With $I_{\circ}(0)=2.48, I_{1}(0)=$ $4 I_{\circ}(0)$ and $I_{2}(0)=20 I_{\circ}(0)-4$, the critical points are given by:

$$
\tilde{g}_{1}^{c}=0.4, \quad \tilde{g}_{2}^{c}=0 ; \quad \tilde{g}_{1}^{c}=0, \quad \tilde{g}_{2}^{c}=0.0055
$$

as indicated 2 in Fig. 1. These critical values are sufficiently small even for $N_{f}=1$.

As for the wave-function renormalization $Z_{2}(p)$ in eq. (23), it depends on the dynamics of the left-handed Weyl fermion $\psi_{L}^{i}$ in this region. By the large- $N_{f}$ calculation at weak-couplings (see appendix II and Figs.4 ,5), we are able to evaluate the wave-function renormalization $Z_{2}(p)$, which is given by

$$
\begin{aligned}
Z_{2}(p) & =1+\frac{2}{N_{f}} \int_{k, q}\left(\tilde{g}_{1}+4 \tilde{g}_{2} w(p-k) w\left(k+\frac{q}{2}\right)\right)^{2} \frac{\sum_{\mu \nu} \gamma_{\mu} \gamma_{\nu} \sin (p-k)^{\mu} \sin p^{\nu}}{\sum_{\lambda \rho} \sin ^{2}(p-k)_{\lambda} \sin ^{2} p_{\rho}} R(k, q) \\
R(k, q) & =\frac{\sum_{\sigma} \sin \left(k-\frac{q}{2}\right)^{\sigma} \sin \left(k+\frac{q}{2}\right)_{\sigma}}{\sum_{\sigma \sigma^{\prime}} \sin ^{2}\left(k-\frac{q}{2}\right)^{\sigma} \sin ^{2}\left(k+\frac{q}{2}\right)^{\sigma^{\prime}}} .
\end{aligned}
$$

This broken phase cannot be a candidate for a real chiral gauge theory (e.g., the Standard Model) for the reasons that $(i) \psi_{L}^{2}$ is doubled (21); (ii) the 
spontaneous symmetry breakdown of the $S U_{L}(2)$ chiral symmetry is caused by the hard breaking Wilson term [16] (20) (dimension-5 operator), which must contribute to the intermediate gauge-boson masses through the perturbative gauge interaction and disposal of Goldstone modes. The intermediate gauge boson masses turn out to be $O\left(\frac{1}{a}\right)$. This, however, is phenomenologically unacceptable.

\section{The strong-coupling region}

We turn to the strong-coupling region, where $g_{1}\left(g_{2}\right)$ are sufficiently larger than a certain critical value $g_{1}^{c}\left(g_{2}^{c}\right)$ (indicated 3 in Fig. 1 ). We can show that the $\psi_{L}^{i}$ and $\psi_{R}$ in (11) are bound to form the three-fermion states

$$
\Psi_{R}^{i}=\frac{1}{2 a}\left(\bar{\psi}_{R} \cdot \psi_{L}^{i}\right) \psi_{R} ; \quad \Psi_{L}^{n}=\frac{1}{2 a}\left(\bar{\psi}_{L}^{i} \cdot \psi_{R}\right) \psi_{L}^{i}
$$

These three-fermion states are Weyl fermions and respectively pair up with $\bar{\psi}_{R}$ and $\bar{\psi}_{L}^{i}$ to be massive, neutral $\Psi_{n}$ and charged $\Psi_{c}^{i}$ Dirac modes,

$$
\Psi_{c}^{i}=\left(\psi_{L}^{i}, \Psi_{R}^{i}\right), \quad \Psi_{n}=\left(\Psi_{L}^{n}, \psi_{R}\right) .
$$

These three-fermion states (39) carry the appropriate quantum numbers of the chiral group that accommodates $\psi_{L}^{i}$ and $\psi_{R} . \Psi_{R}^{i}$ is $S U_{L}(2)$-covariant and $U_{R}(1)$ invariant. $\Psi_{L}^{n}$ is $S U_{L}(2)$-invariant and $U_{R}(1)$-covariant. Thus, the spectrum of the massive composite Dirac fermions $\Psi_{c}^{i}$ and $\Psi_{n}$ is vector-like, consistent with the $S U_{L}(2) \otimes U_{R}(1)$ chiral symmetry.

In order to study 1PI vertex functions containing the external legs of threefermion states (39), we define the composite "primed" fields: the right-handed and charged three-fermion states as

$$
\Psi_{R}^{\prime i} \equiv\left\langle\Psi_{R}^{i}\right\rangle=\frac{1}{2 a} \frac{\delta^{(3)} W(\eta)}{\delta \eta_{R}(x) \delta \bar{\eta}_{L}^{i}(x) \delta \bar{\eta}_{R}(x)} ;
$$

and the left-handed and neutral three-fermion states as

$$
\Psi_{L}^{\prime n} \equiv\left\langle\Psi_{L}^{n}\right\rangle=\frac{1}{2 a} \frac{\delta^{(3)} W(\eta)}{\delta \eta_{L}^{i}(x) \delta \bar{\eta}_{R}(x) \delta \bar{\eta}_{L}^{i}(x)} .
$$

Thus, 1PI vertex functions containing the external legs of three-fermion states (39),

$$
\frac{\delta^{(2)} \Gamma}{\delta \Psi_{R}^{\prime i}(x) \bar{\psi}_{L}^{\prime j}(y)}, \quad \frac{\delta^{(2)} \Gamma}{\delta \Psi_{L}^{\prime n}(x) \bar{\psi}_{R}^{\prime}(y)}, \quad \cdots
$$

are the truncations of the Green functions

$$
\begin{aligned}
\left\langle\Psi_{R}^{i}(x) \bar{\psi}_{L}^{j}(0)\right\rangle & =\frac{1}{2 a} \frac{\delta^{(4)} W(\eta)}{\delta \eta_{R}(x) \delta \bar{\eta}_{L}^{i}(x) \delta \bar{\eta}_{R}(x) \delta \eta_{L}^{j}(0)} \\
\left\langle\Psi_{L}^{n}(x) \bar{\psi}_{R}(0)\right\rangle & =\frac{1}{2 a} \frac{\delta^{(4)} W(\eta)}{\delta \eta_{L}^{i}(x) \delta \bar{\eta}_{R}(x) \delta \bar{\eta}_{L}^{i}(x) \delta \eta_{R}(0)}, \cdots
\end{aligned}
$$


eqs. (43) are the most simple couplings between three-fermion states and elementary fields.

Now, we study the propagators of composite Dirac fermions (40). As for the neutral composite Dirac fermion, its propagator is given as

$\left\langle\Psi_{n}(0) \bar{\Psi}_{n}(x)\right\rangle_{\circ}=\left\langle\Psi_{L}^{n}(0) \bar{\Psi}_{L}^{n}(x)\right\rangle_{\circ}+\left\langle\Psi_{L}^{n}(0) \bar{\psi}_{R}(x)\right\rangle_{\circ}+\left\langle\psi_{R}(0) \bar{\Psi}_{L}^{n}(x)\right\rangle_{\circ}+\left\langle\psi_{R}(0) \bar{\psi}_{R}(x)\right\rangle_{\circ}$.

This propagator can be determined up to a wave renormalization function by the Ward identity of the $\psi_{R}$ shift-symmetry. Taking a functional derivative of the Ward identity eq. (15) with respect to $\Psi_{L}^{\prime n}(x)$ and then putting external sources $\eta=0$ and $J=0$, we can derive

$$
\int_{x} e^{i p x} \frac{\delta^{(2)} \Gamma}{\delta \Psi_{L}^{\prime n}(x) \delta \bar{\psi}_{R}^{\prime}(0)}=\frac{1}{2} M(p)
$$

where

$$
M(p)=2 a\left(g_{1}+4 g_{2} w^{2}(p)\right) .
$$

On the basis of the 1PI vertex functions eqs. (18,46), we can determine the inverse propagator (45) of the neutral composite Dirac fermion $\Psi_{n}(x)$ to be,

$$
S_{n}^{-1}(p)=\frac{i}{a} \sum_{\mu} \gamma_{\mu} \sin p^{\mu} Z_{2}^{n}(p) P_{L}+\frac{i}{a} \sum_{\mu} \gamma_{\mu} \sin p^{\mu} P_{R}+M(p),
$$

where the unknown $Z_{2}^{n}(p)$ is a wave-function renormalization for $\Psi_{L}^{n}(x)$ field.

The propagator of the charged composite Dirac fermion (40) is

$\left\langle\Psi_{c}^{i}(0) \bar{\Psi}_{c}^{j}(x)\right\rangle_{\circ}=\left\langle\psi_{L}^{i}(0) \bar{\psi}_{L}^{j}(x)\right\rangle_{\circ}+\left\langle\Psi_{R}^{i}(0) \bar{\psi}_{L}^{j}(x)\right\rangle_{\circ}+\left\langle\psi_{L}^{i}(0) \bar{\Psi}_{R}^{j}(x)\right\rangle_{\circ}+\left\langle\Psi_{R}^{i}(0) \bar{\Psi}_{R}^{j}(x)\right\rangle_{\circ}$,

which we have to calculate by adopting the strong-coupling expansion. For the purpose of understanding three-fermion bound states, we henceforth focus on the region $\left(g_{1} \gg 1, g_{2}=0\right)$. We make a rescaling of the fermion fields,

$$
\psi_{L}^{i}(x) \rightarrow\left(g_{1}\right)^{\frac{1}{4}} \psi_{L}^{i}(x) ; \quad \psi_{R}(x) \rightarrow\left(g_{1}\right)^{\frac{1}{4}} \psi_{R}(x),
$$

and rewrite the action (11) and partition function in terms of the new fermion fields

$$
\begin{aligned}
S_{f}(x) & =\frac{1}{2 a g_{1}^{\frac{1}{2}}} \sum_{\mu}\left(\bar{\psi}_{L}^{i}(x) \gamma_{\mu} \partial^{\mu} \psi_{L}^{i}(x)+\bar{\psi}_{R}(x) \gamma_{\mu} \partial^{\mu} \psi_{R}(x)\right) \\
S_{1}(x) & =\bar{\psi}_{L}^{i}(x) \cdot \psi_{R}(x) \bar{\psi}_{R}(x) \cdot \psi_{L}^{i}(x) .
\end{aligned}
$$

For the coupling $g_{1} \rightarrow \infty$, the kinetic terms $S_{f}(x)$ can be dropped and we consider this strong-coupling limit. With $S_{2}(x)$ given in eq. (52), the integral of $e^{-S_{2}(x)}$ is

\footnotetext{
${ }^{3}$ I thank Y. Shamir for discussions on these propagators.
} 
given (see eq. (95) with $\Delta(x)=1$ and $g_{2} \rightarrow g_{1}$ in appendix III) by

$$
\begin{aligned}
Z & =\Pi_{x i \alpha} \int\left[d \bar{\psi}_{R}^{\alpha}(x) d \psi_{R}^{\alpha}(x)\right]\left[d \bar{\psi}_{L}^{i \alpha}(x) d \psi_{L}^{i \alpha}(x)\right] \exp \left(-S_{1}(x)\right) \\
& =2^{4 N}
\end{aligned}
$$

where " $N$ " is the number of lattice sites. eq. (53) shows a non-trivial strongcoupling limit.

We now can perform the strong-coupling expansion in powers of $\frac{1}{g_{1}}$ about this strong-coupling limit to calculate eq. (49). To all orders in this expansion, the spectrum of the theory only contains massive states 49,45$)$ even though the $S U_{L}(2) \otimes U_{R}(1)$ chiral symmetry is exact. We define two-point functions in the propagator of the charged Dirac particle (49) to be

$$
\begin{aligned}
S_{L L}^{i j}(x) & \equiv\left\langle\psi_{L}^{i}(0), \bar{\psi}_{L}^{j}(x)\right\rangle \\
S_{M L}^{i j}(x) & \equiv\left\langle\psi_{L}^{i}(0),\left[\bar{\psi}_{L}^{j}(x) \cdot \psi_{R}(x)\right] \bar{\psi}_{R}(x)\right\rangle \\
S_{M M}^{i j}(x) & \equiv\left\langle\left[\bar{\psi}_{R}(0) \cdot \psi_{L}^{i}(0)\right] \psi_{R}(0),\left[\bar{\psi}_{L}^{j}(x) \cdot \psi_{R}(x)\right] \bar{\psi}_{R}(x)\right\rangle .
\end{aligned}
$$

We compute these two-point functions by the strong-coupling expansion in powers of $O\left(\frac{1}{g_{1}}\right)$. Using relations (152,154) with $\Delta(x)=1$ and $g_{2} \rightarrow g_{1}$ in appendix III, in the lowest non-trivial order, we obtain the following recursion relations

$$
\begin{aligned}
S_{L L}^{i j}(x) & =\frac{1}{g_{1}}\left(\frac{1}{2 a}\right)^{3} \sum_{\mu}^{\dagger} S_{M L}^{i j}(x+\mu) \gamma_{\mu}, \\
S_{M L}^{i j}(x) & =\frac{\delta(x) \delta_{i j}}{2 g_{1}}+\frac{1}{g_{1}}\left(\frac{1}{2 a}\right) \sum_{\mu}^{\dagger} S_{L L}^{i j}(x+\mu) \gamma_{\mu} . \\
S_{M M}^{i j}(x) & =\frac{1}{g_{1}}\left(\frac{1}{2 a}\right) \sum_{\mu}^{\dagger} \gamma_{\mu} \gamma_{\circ} S_{M L}^{i j \dagger}(x+\mu) \gamma_{\circ},
\end{aligned}
$$

where for an arbitrary function $f(x)$,

$$
\sum_{\mu}^{\dagger} f(x)=\sum_{\mu}(f(x+\mu)-f(x-\mu)) .
$$

Transforming these two-point functions (54,55,56) into momentum space, $(X=$ $L L, M L, M M$ respectively)

$$
S_{X}^{i j}(p)=\int d^{4} x e^{-i p x} S_{X}^{i j}(x)
$$

one gets three recursion relations in momentum space

$$
\begin{aligned}
S_{L L}^{i j}(p) & =\frac{1}{g_{1}}\left(\frac{i}{4 a^{3}}\right) \sum_{\mu} \sin p^{\mu} S_{M L}^{i j}(p) \gamma_{\mu}, \\
S_{M L}^{i j}(p) & =\frac{\delta_{i j}}{2 g_{1}}+\frac{i}{g_{1} a} \sum_{\mu} \sin p^{\mu} S_{L L}^{i j}(p) \gamma_{\mu} . \\
S_{M M}^{i j}(p) & =\frac{1}{g_{1}}\left(\frac{i}{a}\right) \sum_{\mu} \sin p^{\mu} \gamma_{\mu} \gamma_{\circ} S_{M L}^{i j \dagger}(p) \gamma_{\circ} .
\end{aligned}
$$


We solve these recursion relations (62,63, 64) and obtain

$$
\begin{aligned}
S_{L L}^{i j}(p) & =P_{L} \frac{\delta_{i j} \frac{i}{2 a} \sum_{\mu} \sin p^{\mu} \gamma_{\mu}}{\frac{1}{a^{2}} \sum_{\mu} \sin ^{2} p_{\mu}+M_{1}^{2}} P_{R} \\
\frac{1}{2 a} S_{M L}^{i j}(p) & =P_{L} \frac{\delta_{i j} \frac{1}{2} M(p)}{\frac{1}{a^{2}} \sum_{\mu} \sin ^{2} p_{\mu}+M_{1}^{2}} P_{L} \\
\left(\frac{1}{2 a}\right)^{2} S_{M M}^{i j}(p) & =P_{R} \frac{\delta_{i j} \frac{i}{2 a} \sum_{\mu} \sin p^{\mu} \gamma_{\mu}}{\frac{1}{a^{2}} \sum_{\mu} \sin ^{2} p_{\mu}+M_{1}^{2}} P_{L},
\end{aligned}
$$

where the chiral-invariant mass is

$$
M_{1}=2 g_{1} a \text {. }
$$

The second two-point function in eq. (49) is given by,

$$
\frac{1}{2 a}\left\langle\left[\bar{\psi}_{R}(x) \cdot \psi_{L}^{j}(x)\right] \psi_{R}(x), \bar{\psi}_{L}^{i}(0)\right\rangle=\frac{1}{2 a} \gamma_{\circ} S_{M L}^{\dagger i j}(x) \gamma_{\circ}=P_{R} \frac{\delta_{i j} \frac{1}{2} M_{1}}{\frac{1}{a^{2}} \sum_{\mu} \sin ^{2} p_{\mu}+M_{1}^{2}} P_{R} .
$$

We substitute eqs. (65,66,67,69) into eq. (49), in the lowest non-trivial order of the strong-coupling expansion and obtain the massive propagator of the charged Dirac fermions $\Psi_{c}^{i}$,

$$
S_{c}^{i j}(p)=\int d^{4} x e^{-i p x}\left\langle\Psi_{c}^{i}(0) \bar{\Psi}_{c}^{j}(x)\right\rangle=\delta_{i j} \frac{\frac{i}{a} \sum_{\mu} \sin p^{\mu} \gamma_{\mu}+M_{1}}{\frac{1}{a^{2}} \sum_{\mu} \sin ^{2} p_{\mu}+M_{1}^{2}} .
$$

Analogously, the massive propagator (45) of the neutral Dirac fermions $\Psi_{n}$ can be calculated in the same way

$$
S_{n}(p)=\int d^{4} x e^{-i p x}\left\langle\Psi_{n}(0) \bar{\Psi}_{n}(x)\right\rangle=\frac{\frac{i}{a} \sum_{\mu} \sin p^{\mu} \gamma_{\mu}+M_{1}}{\frac{1}{a^{2}} \sum_{\mu} \sin ^{2} p_{\mu}+M_{1}^{2}}
$$

which coincides, for $g_{2}=0$ and $Z_{2}^{n}(p)=1$, with eq. (48) that is derived by using the Ward identity (15) of the $\psi_{R}(x)$ shift-symmetry. eqs. (70,71) show that the spectrum is vector-like and massive, consistent with the $S U_{L}(2) \otimes U_{R}(1)$ chiral symmetry. In this strong coupling symmetric phase, all fermion modes including doublers and normal modes of $\psi_{L}^{i}(x)$ and $\psi_{R}(x)$ are bound to be three-fermion states and then form massive Dirac fermion states. The spectrum of normal modes and doublers is massive and vector-like. This is certainly not what we desire.

\section{Wedges: different thresholds of forming three- fermion states}

The three-fermion states (39) are composed of three elementary Weyl modes through the multifermion couplings $S_{1}(x)$ and $S_{2}(x)$ (1). As expected by Eichten 
and Preskill [2], due to the fact that the multifermion coupling $S_{2}(x)$ gives different contributions to the effective value of $g_{1}$ at large distances for the sixteen Weyl modes of $\psi_{L}^{i}$ and $\psi_{R}$ in the action (1), these sixteen modes have different thresholds $g_{1}^{c}\left(g_{2}^{c}\right)$ of forming three-fermion states. In fact, we can explicitly see this point by looking at the four-fermion 1PI vertex function (27), which is exactly obtained by the Ward identity (15) of the $\psi_{R}(x)$ shift-symmetry,

$$
\Gamma^{(4)}\left(p, p^{\prime}, q\right)=g_{1}+4 g_{2} w\left(p+\frac{q}{2}\right) w\left(p^{\prime}+\frac{q}{2}\right),
$$

where $p+\frac{q}{2}$ and $p^{\prime}+\frac{q}{2}$ are momenta of $\psi_{R}(x)$ field; $p-\frac{q}{2}$ and $p^{\prime}-\frac{q}{2}$ are momenta of $\psi_{L}^{i}(x)$ field ( $q$ is the momentum transfer as shown in Fig. 2). In the case of $g_{1}=0$, the multifermion coupling associated with the normal modes of $\psi_{R}(x)$ and $\psi_{L}^{i}(x)$ is very small $\left(O\left(a^{2}\right)\right)$.

These different thresholds $g_{1}^{c}\left(g_{2}^{c}\right)$ can be qualitatively determined by the following discussion. We consider a complex composite field,

$$
\mathcal{A}^{i}=\bar{\psi}_{R} \cdot \psi_{L}^{i}
$$

and its real and imaginary parts are four composite scalars $(i=1,2)$

$$
\begin{aligned}
A_{1}^{i} & =\frac{1}{2}\left(\bar{\psi}_{L}^{i} \cdot \psi_{R}+\bar{\psi}_{R} \cdot \psi_{L}^{i}\right) \\
A_{2}^{i} & =\frac{i}{2}\left(\bar{\psi}_{L}^{i} \cdot \psi_{R}-\bar{\psi}_{R} \cdot \psi_{L}^{i}\right) .
\end{aligned}
$$

These composite scalars and their propagators are determined by the two-point function of the theory,

$$
\begin{aligned}
G^{i j}(x) & =\left\langle\mathcal{A}^{i}(0), \mathcal{A}^{\dagger j}(x)\right\rangle \\
& =\left\langle\bar{\psi}_{R}(0) \cdot \psi_{L}^{i}(0), \bar{\psi}_{R}(x) \cdot \psi_{L}^{j}(x)\right\rangle .
\end{aligned}
$$

For simplicity, we put $g_{2}=0$ and eq. (72) becomes

$$
\Gamma^{(4)}\left(p, p^{\prime}, q\right)=g_{1}
$$

Adopting the strong-coupling expansion in powers of $\frac{1}{g_{1}}\left(g_{1} \gg 1\right)$ and the relation (157) with $\Delta(x)=1$ and $g_{2} \rightarrow g_{1}$ in appendix III, we obtain the following recursion relation

$$
G^{i j}(x)=\frac{\delta(x) \delta_{i j}}{g_{1}}+\frac{1}{g_{1}}\left(\frac{1}{2 a}\right)^{2} \sum_{ \pm \mu} G^{i j}(x+\mu) .
$$

Going to momentum space, we have

$$
G^{i j}(q)=\int d^{4} x e^{-i q x} G^{i j}(x)
$$


where $q$ is the momentum of the composite scalar $\mathcal{A}^{i}=\bar{\psi}_{R} \cdot \psi_{L}^{i}$. The recursion relation $(77)$ in momentum space is given by

$$
G^{i j}(q)=\frac{\delta_{i j}}{g_{1}}+\left(\frac{1}{2 a^{2}}\right) \frac{1}{g_{1}} \sum_{ \pm \mu} \cos q_{\mu} G^{i j}(q)
$$

As a result, we find the propagators for these four massive composite scalar modes of $\mathcal{A}^{i}=\bar{\psi}_{R} \cdot \psi_{L}^{i}$,

$$
\begin{aligned}
G^{i j}(q) & =4 \frac{\delta_{i j}}{\frac{4}{a^{2}} \sum_{\mu} \sin ^{2} \frac{q_{\mu}}{2}+\mu^{2}} \\
\mu^{2} & =4\left(g_{1}-\frac{2}{a^{2}}\right)
\end{aligned}
$$

which are degenerate owing to the exact $S U_{L}(2) \otimes U_{R}(1)$ chiral symmetry. Thus,

$$
\mu^{2} \mathcal{A}^{i} \mathcal{A}^{i \dagger}
$$

effectively gives the mass term of the composite scalar field $\mathcal{A}^{i}$ in the effective Lagrangian. We assume that the 1 PI vertex $\mathcal{A}^{j} \mathcal{A}^{\dagger j} \mathcal{A}^{i} \mathcal{A}^{\dagger i}$ is positive and the energy of ground states of the theory is bound from the bellow. Then, we can qualitatively discuss [3] the second order phase transition (threshold) from the strong coupling symmetric phase to the weak-coupling NJL broken phase by examining the mass term of these composite scalars (82). Spontaneous symmetry breaking $S U(2) \rightarrow U(1)$ occurs, where $\mu^{2}>0$ turns to $\mu^{2}<0$. eq. (81) for $\mu^{2}=0$ gives rise to the critical point:

$$
g_{1}^{c} a^{2}=2, \quad g_{2}=0
$$

(as indicated in Fig. 1) where a phase transition takes place between the NJL symmetry-breaking phase and the strong-coupling symmetric phase.

As for the case $g_{2} \neq 0$, the second multifermion coupling in eq. (72) has to be taken into account. We should not doubt that the thresholds $g_{1}^{c}\left(g_{2}^{c}\right)$ depend on the sixteen modes of $\psi_{L}^{i}$ and $\psi_{R}$. In order to see this phenomenon, we can effectively replace the coupling $g_{1}$ in eq. (81) by the coupling (72) involving $g_{2}$. As a result, the thresholds of binding three-fermion states can be qualitatively determined by

$$
\mu^{2}=4\left(g_{1}+4 g_{2} w\left(p+\frac{q}{2}\right) w\left(p^{\prime}+\frac{q}{2}\right)-\frac{2}{a^{2}}\right)=0 .
$$

Let us first consider the multifermion couplings of each mode " $p$ " of the $\psi_{L}^{i}$ and $\psi_{R}$, namely, we set $p=p^{\prime}, q \ll 1$ in the four-point vertex (72). We obtain

$$
\mu^{2}=4\left(g_{1}+4 g_{2} w^{2}(p)-\frac{2}{a^{2}}\right) .
$$


Thus, $\mu^{2}=0$ gives rise to the critical lines (thresholds):

$$
g_{1}^{c} a^{2}=2, g_{2}=0 ; \quad g_{1}=0, a^{2} g_{2}^{c, b}=0.008,
$$

where the first binding threshold of the doubler $p=(\pi, \pi, \pi, \pi)$ is located, and

$$
g_{1}^{c} a^{2}=2, g_{2}=0 ; \quad g_{1}=0, a^{2} g_{2}^{c, a}=0.124,
$$

where the last binding threshold of the doublers $p=(\pi, 0,0,0)$ is located. In between (indicated 4 in Fig. 1) there are the binding thresholds of the doublers $p=(\pi, \pi, 0,0)$ and $p=(\pi, \pi, \pi, 0)$ in eq. (85), and the binding thresholds of the different doublers $p \neq p^{\prime}$ in eq. (84). Above $g_{1}^{c, a}$ all doublers are supposed to be bound. As for the normal modes $(\tilde{p})$ of $\psi_{L}^{i}$ and $\psi_{R}$, when $g_{1} \ll 1$, the multifermion coupling (72), $\Gamma^{(4)}=g_{1}+4 g_{2} w^{2}(\tilde{p})$, is no longer strong enough to form the threefermion states $\left(\bar{\psi}_{L}^{i} \cdot \psi_{R}\right) \psi_{L}^{i}$ and $\left(\bar{\psi}_{R} \cdot \psi_{L}^{i}\right) \psi_{R}$ unless $a^{2} g_{2} \rightarrow \infty$. It is conceivable that the threshold for binding normal modes, which is given by eq. (85) for $p=\tilde{p}$,

$$
g_{1}+a g_{2} O\left(\tilde{p}^{2}\right)-\frac{2}{a^{2}}=0
$$

analytically continues to the limit

$$
g_{2}^{c, \infty} \rightarrow \infty, \quad g_{1} \rightarrow 0
$$

as indicated in Fig. 1. Certainly, these thresholds cannot be regarded as quantitative results obtained by precise computations.

Thus, as expected in ref. [2], several wedges open up as $g_{1}, g_{2}$ increase in the NJL phase (indicated 5 in Fig. 1), in between the critical lines along which three-fermion states of normal modes and doublers of $\psi_{L}^{i}$ and $\psi_{R}$ respectively approach their thresholds. In the initial part of the NJL phase (the deep NJL broken phase), the normal modes and doublers of the $\psi_{L}^{i}$ and $\psi_{R}$ undergo the NJL phenomenon and contribute to eqs. (20,21), as discussed in section 2. As $g_{1}, g_{2}$ increase, all these modes, one after another, gradually disassociate from the NJL phenomenon and no longer contribute to eqs. (20,21). Instead, they turn to associate with the EP phenomenon and contribute to eqs. (70,71). The first and last doublers of $\psi_{L}^{i}$ and $\psi_{R}$ making this transition are $p=(\pi, \pi, \pi, \pi)$ (86) and $p=(\pi, 0,0,0)$ (87) respectively. At the end of this sequence, normal modes $(p=\tilde{p})$ make this transition (89), due to the fact that they possess the different effective multifermion coupling (72). There are two possibilities that one might expect to have the continuum limit of chiral fermions defined either on one of these thresholds or within one of these wedges.

Had these thresholds separated the two symmetric phases, (strong-couplings and the weak-coupling symmetric phases) we would have found a threshold over which an EP phase transition takes place, namely, the massless normal modes of the $\psi_{L}^{i}$ and $\psi_{R}$ are becoming massive, while the doublers of $\psi_{L}^{i}$ and $\psi_{R}$ have 
acquired chiral-invariant masses and decoupled (70,771). We would define a continuum theory of massless free chiral fermions [2] on such a threshold. However, this is not the real case [3] for $g_{2}=0$, as has been seen in eq. (81), $\mu^{2}>0$ turning to $\mu^{2}<0$ at $g_{1}=g_{1}^{c}=2$ indicates a phase transition between the strong-coupling symmetric phase and the spontaneous symmetry breaking phase, which separates the strong-coupling and weak coupling symmetric phases. Namely, there is no EP phase transition taking place over any one of those thresholds. This can be clearly seen (as shown in Fig. 1) by comparison of the thresholds of three-fermion states (83,86,87) and the NJL phase transition (37).

Had one of these wedges contained a spectrum in which all doublers of $\psi_{L}^{i}$ and $\psi_{R}$ decouple as Dirac fermions by acquiring chiral-invariant masses (70,71) and the normal modes of $\psi_{L}^{i}$ and $\psi_{R}$ remain massless and free, and within that wedge we would have obtained a continuous theory of massless free chiral fermions [2]. However, this seems not really the case for the reason that all these wedges from the deep NJL broken phase to the deep EP symmetric phase are continuously connected. These thresholds and wedges qualitatively determined cannot be considered to be very distinct for the spectrum of the theory, since there may be the coexistence of less tightly bound three-fermion states of doublers and unbound doublers, the possible strong fluctuations of these three-fermion states and mixing between different modes.

Even within the last wedge, as indicated 5 in Fig. 1, if we assume that there is a such region where the couplings $g_{1}$ and $g_{2}$ are sufficiently larger than the threshold (87) where all doublers are supposed to be strongly bound 6 , we should have the undoubled low-energy spectrum that involves only the normal modes of $\psi_{L}^{i}$ and $\psi_{R}$. However, because of the multifermion coupling $g_{1} \neq 0$, these normal modes of $\psi_{L}^{i}$ and $\psi_{R}$ still remain in the NJL broken phase, the $S U_{L}(2)$ chiral symmetry is still violated by $\Sigma^{1}(0)=\rho v^{1}(30)$, to which only normal modes contribute. The propagators of the normal modes in this wedge should be the same as eqs. (20,21) for $p=\tilde{p}$. Due to the fact that chiral gauge symmetry is broken by the NJL phenomenon associated with the normal modes, the propagator of gauge bosons will not have the correct properties. Furthermore, when $g_{1} \neq 0$, the normal mode of $\psi_{R}$ is not guaranteed to completely decouple from that of $\psi_{L}^{i}$.

So far, we have almost no possibility to find a distinct threshold for the secondorder phase transition to define the continuum limit for chiral fermions and a distinctly chiral-symmetric region in the phase space where a desired spectrum of chiral fermions exists. Nevertheless, a possible resolution of this undesirable situation could probably take place in a particular symmetric phase that is a segment in the phase diagram, where the doublers of $\psi_{L}^{i}$ and $\psi_{R}$ have formed three-fermion states $\left(\bar{\psi}_{R} \cdot \psi_{L}^{i}\right) \psi_{R}$ and $\left(\bar{\psi}_{L}^{i} \cdot \psi_{R}\right) \psi_{L}^{i}$ via the EP phenomenon, while the normal modes of $\psi_{L}^{i}$ and $\psi_{R}$ have neither formed such bound states yet and

\footnotetext{
${ }^{4}$ Here, by the notion of strongly bound, we mean that all doublers are completely and tightly bound into three-fermion states and no doublers are left in the spectrum.
} 
nor are they associated with the NJL-phenomenon. If this case happened, we might find a scaling region of the continuum limit of lattice chiral fermions.

\section{A possible scaling region of chiral fermions}

The possible scaling region of the continuum limit of lattice chiral fermion can probably be found once we go onto the line where $g_{1}=0$ and $g_{2}$ is in a certain segment A indicated in Fig. 1. Assuming that above $g_{2}^{c, a}$ all doublers are strongly bound, above $g_{2}^{c, \infty}$ all modes are strongly bound and $g_{2}^{c, \infty}>g_{2}^{c, a} \gg 1$, we give the definition of this peculiar segment $A$ :

$$
A=\left[g_{1}=0, g_{2}^{c, a}<g_{2}<g_{2}^{c, \infty}\right] .
$$

In order to show this segment could be a possible candidate for the scaling region of the continuum limit of lattice chiral fermions, we are bound to demonstrate the following properties of the theory in this segment:

1. the normal mode of $\psi_{R}$ is a free mode and decoupled;

2. no spontaneous chiral symmetry breaking occurs ;

3. all doublers are strongly bound to be massive Dirac fermions and decoupled consistently with chiral symmetry;

4. the normal modes of $\psi_{L}^{i}(x)$ and $\psi_{R}(x)$ have not yet been bound to the three-fermion state $\left(\bar{\psi}_{R} \cdot \psi_{L}^{i}\right) \psi_{R}$, an undoubled chiral mode of $\psi_{L}^{i}(x)$ exists in the low-energy spectrum.

We have already discussed the first property in section 2 . In the case of $g_{1}=0$, the normal mode of $\psi_{R}(x)$ is massless and does not receive the wavefunction renormalization,

$$
S_{R R}^{-1}=i \gamma_{\mu} \tilde{p}^{\mu} .
$$

Furthermore, in section 4 based on the Ward identity (15) associated with the $\psi_{R}(x)$ shift-symmetry, we have determined the propagator of the neutral Dirac particle (48), which is made up of the $\psi_{R}(x)$ and its left-handed composite partner $\Psi_{L}^{n}(x)(39)$, up to a wave function renormalization for $\Psi_{L}^{n}(x)$ field.

As for the other properties, since $g_{1}=0, g_{2} \gg 1$ in this segment, we can adopt the strong-coupling expansion in powers of $\frac{1}{g_{2}}$ to calculate 1PI functions that describe those properties of the theory. Thus, we make a rescaling of the fermion fields,

$$
\psi_{L}^{i}(x) \rightarrow\left(g_{2}\right)^{\frac{1}{4}} \psi_{L}^{i}(x) ; \quad \psi_{R}(x) \rightarrow\left(g_{2}\right)^{\frac{1}{4}} \psi_{R}(x),
$$


and rewrite the action (11) and partition function in terms of new fermion fields

$$
\begin{aligned}
S_{f}(x) & =\frac{1}{2 a g_{2}^{\frac{1}{2}}} \sum_{\mu}\left(\bar{\psi}_{L}^{i}(x) \gamma_{\mu} \partial^{\mu} \psi_{L}^{i}(x)+\bar{\psi}_{R}(x) \gamma_{\mu} \partial^{\mu} \psi_{R}(x)\right) \\
S_{2}(x) & =\bar{\psi}_{L}^{i}(x) \cdot\left[\Delta \psi_{R}(x)\right]\left[\Delta \bar{\psi}_{R}(x)\right] \cdot \psi_{L}^{i}(x) .
\end{aligned}
$$

For the coupling $g_{2} \rightarrow \infty$, the kinetic terms $S_{f}(x)$ can be dropped and we consider this strong-coupling limit. With $S_{2}(x)$ given in eq. (94), the integral of $e^{-S_{2}(x)}$ can be computed (see the beginning of appendix III)

$$
\begin{aligned}
Z & =\Pi_{x i \alpha} \int\left[d \bar{\psi}_{R}^{\alpha}(x) d \psi_{R}^{\alpha}(x)\right]\left[d \bar{\psi}_{L}^{i \alpha}(x) d \psi_{L}^{i \alpha}(x)\right] \exp \left(-S_{2}(x)\right) \\
& =2^{4 N}\left(\operatorname{det} \Delta^{2}(x)\right)^{4}
\end{aligned}
$$

where the determent is taken only over the lattice space-time and $N$ is the number of lattice sites. For the non-zero eigenvalues of the operator $\Delta^{2}(x)$, eq. (95) shows an existence of the sensible strong-coupling limit. However, as for the zero eigenvalue of the operator $\Delta^{2}(x)$, this strong-coupling limit is trivial and should not be analytic and the strong-coupling expansion in powers of $\frac{1}{g_{2}}$ breaks down.

We consider the second property that this segment is entirely symmetric. The vanishing of the 1PI self-energy function $\Sigma(p)(19)$ indicates no spontaneous symmetry breaking of the theory. In sections 2 and 3, on the basis of both the Ward identity (25) of the $\psi_{R}$ shift-symmetry and the explicit computation in the large- $N_{f}$ weak-coupling expansion (33), we show that this 1PI self-energy function $\Sigma(p)$ vanishes at zero momentum, provided $g_{1}=0$,

$$
\Sigma(p)=0, \quad p=0 .
$$

Obviously, we need to show that this 1PI self-energy function $\Sigma(p)$ vanishes for $p \neq 0$ in this segment as well. For this purpose, we have to calculate the following two-point functions

$$
\begin{aligned}
S_{R L}^{j}(x) & \equiv\left\langle\psi_{R}(0), \bar{\psi}_{L}^{j}(x)\right\rangle, \\
S_{M R}^{j}(x) & \equiv\left\langle\psi_{R}(0),\left[\bar{\psi}_{L}^{j}(x) \cdot \psi_{R}(x)\right] \bar{\psi}_{R}(x)\right\rangle .
\end{aligned}
$$

In appendix III, using the strong-coupling expansion in powers $O\left(\frac{1}{g_{2}}\right)$ above the non-trivial strong-coupling limit (95) for the non-zero eigenvalues of $\Delta^{2}(x)$, we obtain the relations (152,154). Armed with these relations (152,154), in the lowest non-trivial order, one gets the following recursion relations

$$
\begin{aligned}
S_{R L}^{j}(x) & =\frac{1}{g_{2} \Delta^{2}(x)}\left(\frac{1}{2 a}\right)^{3} \sum_{\mu}^{\dagger} S_{M R}^{j}(x+\mu) \gamma_{\mu}, \\
S_{M R}^{j}(x) & =\frac{1}{g_{2} \Delta^{2}(x)}\left(\frac{1}{2 a}\right) \sum_{\mu}^{\dagger} S_{R L}^{j}(x+\mu) \gamma_{\mu} .
\end{aligned}
$$


The Fourier transformations of these two-point functions $(97,98)$ into momentum space are

$$
\begin{aligned}
S_{R L}^{j}(p) & =\int d^{4} x e^{-i p x} S_{R L}^{j}(x) \\
S_{M R}^{j}(p) & =\int d^{4} x e^{-i p x} S_{M R}^{j}(x) .
\end{aligned}
$$

For $p \neq 0$ and the non-zero eigenvalue $\Delta(p)=2 w(p) \neq 0(? ?)$, one gets two recursion relations in momentum space,

$$
\begin{aligned}
S_{R L}^{j}(p) & =\frac{1}{4 g_{2} w^{2}(p)}\left(\frac{i}{4 a^{3}}\right) \sum_{\mu} \sin p^{\mu} S_{M R}^{j}(p) \gamma_{\mu} \\
S_{M R}^{j}(p) & =\frac{1}{g w^{2}(p)}\left(\frac{i}{4 a}\right) \sum_{\mu} \sin p^{\mu} S_{R L}^{j}(p) \gamma_{\mu} .
\end{aligned}
$$

The solution to these recursion relations is

$$
\left(\left(8 a g_{2} w^{2}(p)\right)^{2}+\frac{1}{a^{2}} \sum_{\mu} \sin ^{2} p_{\mu}\right) S_{R L}^{j}(p)=0 .
$$

For $p \neq 0$, clearly we must have

$$
S_{R L}^{j}(p)=0, \quad S_{M R}^{j}(p)=0 .
$$

Similarly, we can prove the vanishing of the following two point functions

$$
\begin{aligned}
& \left\langle\left[\bar{\psi}_{L}^{i}(0) \cdot \psi_{R}(0)\right] \psi_{L}^{i}(0), \bar{\psi}_{L}^{j}(x)\right\rangle, \\
& \left\langle\left[\bar{\psi}_{L}^{i}(0) \cdot \psi_{R}(0)\right] \psi_{L}^{i}(0),\left[\bar{\psi}_{L}^{j}(x) \cdot \psi_{R}(x)\right] \bar{\psi}_{R}(x)\right\rangle .
\end{aligned}
$$

This demonstration can be straightforwardly generalized to show the vanishing of all n-point Green functions that are not $S U_{L}(2) \otimes U_{R}(1)$ chirally symmetric. As a consequence, the segment is entirely symmetric and no spontaneous symmetry breaking takes place.

Now we turn to discussions of the third properties that all doublers are decoupled as massive Dirac fermions consistently with chiral symmetry. Analogously to the case $g_{1} \gg 1, g_{2}=0$ in section 4 , we need to compute the two-point functions (54,55,56) in the propagator of the charged Dirac fermion (49). Performing a strong-coupling expansion in powers of $\frac{1}{g_{2}}$ above the non-trivial strong-coupling limit (95) for non-zero eigenvalues of $\Delta^{2}(x)$, we compute these two-point functions. Armed with the relations (152), (154) and (155) that are obtained in appendix III, in the lowest non-trivial order, we obtain following recursion relations

$$
S_{L L}^{i j}(x)=\frac{1}{g_{2} \Delta^{2}(x)}\left(\frac{1}{2 a}\right)^{3} \sum_{\mu}^{\dagger} S_{M L}^{i j}(x+\mu) \gamma_{\mu}
$$




$$
\begin{aligned}
S_{M L}^{i j}(x) & =\frac{\delta(x) \delta_{i j}}{2 g_{2} \Delta^{2}(x)}+\frac{1}{g_{2} \Delta^{2}(x)}\left(\frac{1}{2 a}\right) \sum_{\mu}^{\dagger} S_{L L}^{i j}(x+\mu) \gamma_{\mu} . \\
S_{M M}^{i j}(x) & =\frac{1}{g_{2} \Delta^{2}(x)}\left(\frac{1}{2 a}\right) \sum_{\mu}^{\dagger} \gamma_{\mu} \gamma_{\circ} S_{M L}^{i j \dagger}(x+\mu) \gamma_{\circ} .
\end{aligned}
$$

Upon the non-trivial strong-coupling limit (95) of $p \neq 0$ and $\Delta(p)=2 w^{2}(p) \neq 0$, Fourier transformation (61) casts three recursion relations in momentum space,

$$
\begin{aligned}
S_{L L}^{i j}(p) & =\frac{1}{4 g_{2} w^{2}(p)}\left(\frac{i}{4 a^{3}}\right) \sum_{\mu} \sin p^{\mu} S_{M L}^{i j}(p) \gamma_{\mu}, \\
S_{M L}^{i j}(p) & =\frac{\delta_{i j}}{8 g_{2} w^{2}(p)}+\frac{i}{4 g_{2} w^{2}(p) a} \sum_{\mu} \sin p^{\mu} S_{L L}^{i j}(p) \gamma_{\mu} . \\
S_{M M}^{i j}(p) & =\frac{1}{4 g_{2} w^{2}(p)}\left(\frac{i}{a}\right) \sum_{\mu} \sin p^{\mu} \gamma_{\mu} \gamma_{\circ} S_{M L}^{i j \dagger}(p) \gamma_{\circ} .
\end{aligned}
$$

We solve these recursion relations (108, 109, 110) and obtain

$$
\begin{aligned}
S_{L L}^{i j}(p) & =P_{L} \frac{\delta_{i j} \frac{i}{2 a} \sum_{\mu} \sin p^{\mu} \gamma_{\mu}}{\frac{1}{a^{2}} \sum_{\mu} \sin ^{2} p_{\mu}+M_{2}^{2}(p)} P_{R} \\
\frac{1}{2 a} S_{M L}^{i j}(p) & =P_{L} \frac{\delta_{i j} \frac{1}{2} M_{2}(p)}{\frac{1}{a^{2}} \sum_{\mu} \sin ^{2} p_{\mu}+M_{2}^{2}(p)} P_{L} \\
\left(\frac{1}{2 a}\right)^{2} S_{M M}^{i j}(p) & =P_{R} \frac{\delta_{i j} \frac{i}{2 a} \sum_{\mu} \sin p^{\mu} \gamma_{\mu}}{\frac{1}{a^{2}} \sum_{\mu} \sin ^{2} p_{\mu}+M_{2}^{2}(p)} P_{L}
\end{aligned}
$$

where the chiral-invariant mass is given as

$$
M_{2}(p)=8 g_{2} a w^{2}(p), \quad p \neq 0
$$

The second two-point function in eq. (49) is given by,

$$
\frac{1}{2 a}\left\langle\left[\bar{\psi}_{R}(x) \cdot \psi_{L}^{j}(x)\right] \psi_{R}(x), \bar{\psi}_{L}^{i}(0)\right\rangle=\frac{1}{2 a} \gamma_{\circ} S_{M L}^{\dagger i j}(x) \gamma_{\circ}=P_{R} \frac{\delta_{i j} \frac{1}{2} M_{2}(p)}{\frac{1}{a^{2}} \sum_{\mu} \sin ^{2} p_{\mu}+M_{2}^{2}(p)} P_{R} .
$$

We substitute eqs. (111,112,113,115) into eq. (49) and obtain the propagator of the charged Dirac doublers $\Psi_{c}^{i}(p \neq 0)$,

$$
S_{c}^{i j}(p)=\int d^{4} x e^{-i p x}\left\langle\Psi_{c}^{i}(0) \bar{\Psi}_{c}^{j}(x)\right\rangle=\delta_{i j} \frac{\frac{i}{a} \sum_{\mu} \sin p^{\mu} \gamma_{\mu}+M_{2}(p)}{\frac{1}{a^{2}} \sum_{\mu} \sin ^{2} p_{\mu}+M_{2}^{2}(p)}
$$

Since $w^{2}(p) \neq 0$ and $M_{2}(p) \neq 0$ for doublers $p=\tilde{p}+\pi_{A}$, this shows that all $S U_{L}(2)$ charged doublers are decoupled as massive Dirac fermions. The massive spectrum for these doublers turns out to be $S U(2)$-QCD vector-like, however, is in consistent agreement with the $S U_{L}(2) \otimes U_{R}(1)$ chiral symmetry. 
Analogously, in the lowest non-trivial order of the strong-coupling expansion in powers of $\frac{1}{g_{2}}$, one gets for $p \neq 0$ and $\Delta(p)=2 w(p) \neq 0$

$$
\begin{aligned}
S_{R R}(p) & =P_{L} \frac{\frac{i}{2 a} \sum_{\mu} \sin p^{\mu} \gamma_{\mu}}{\frac{1}{a^{2}} \sum_{\mu} \sin ^{2} p_{\mu}+M_{2}^{2}(p)} P_{R}, \\
\frac{1}{2 a} S_{M R}(p) & =P_{L} \frac{\frac{1}{2} M_{2}(p)}{\frac{1}{a^{2}} \sum_{\mu} \sin ^{2} p_{\mu}+M_{2}^{2}(p)} P_{L}, \\
\left(\frac{1}{2 a}\right)^{2} S_{M M}(p) & =P_{R} \frac{\frac{i}{2 a} \sum_{\mu} \sin p^{\mu} \gamma_{\mu}}{\frac{1}{a^{2}} \sum_{\mu} \sin ^{2} p_{\mu}+M_{2}^{2}(p)} P_{L},
\end{aligned}
$$

where $S_{R R}(p), S_{M R}(p)$ and $S_{M M}(p)$ are the Fourier transformation of the twopoint functions in the neutral Dirac fermion propagator (45). As a result, the propagator (45) of the neutral Dirac doublers $\Psi_{n}(p \neq 0)$ is given by

$$
S_{n}(p)=\int d^{4} x e^{-i p x}\left\langle\Psi_{n}(0) \bar{\Psi}_{n}(x)\right\rangle=\frac{\frac{i}{a} \sum_{\mu} \sin p^{\mu} \gamma_{\mu}+M_{2}(p)}{\frac{1}{a^{2}} \sum_{\mu} \sin ^{2} p_{\mu}+M_{2}^{2}(p)},
$$

which coincides, for $g_{1}=0$ and $Z_{2}^{n}(p)=1(p \neq 0)$, with eq. (48), which is derived by using the Ward identity (15) of the $\psi_{R}(x)$ shift-symmetry. eq. (120) shows that the spectrum for doublers of the neutral Dirac fermion is vector-like and massive, consistent with the $S U_{L}(2) \otimes U_{R}(1)$ chiral symmetry.

Up to now, we are left with the last, but most important property that the normal modes of the $\psi_{L}^{i}(x)$ and $\psi_{R}(x)$ are massless and chiral in this segment. It is most difficult to have a convincing proof of this property for the time being, since for these normal modes $(p=\tilde{p}=0, \Delta(\tilde{p})=0$ in eq. (95)), a sensible nontrivial strong-coupling limit does not exist and the strong-coupling expansion in powers of $\frac{1}{g_{2}}$ fails to converge analytically. We are actually not able to compute the spectra (propagators) of these normal modes to see whether or not they are chiral in this segment. As a consequence of the interacting action (11) presented in this paper being local, the effective action (inverse propagator) that is bilinear in terms of interpolating fields should be local and analytical in the whole Brillouin zone (this statement has not received a complete proof [17] for the entire range of interacting strength), thus, the "no-go" theorem of Nielsen and Ninomiya is still applicable to this case [9]. Based on this observation, one might argue that the massless spectrum of normal modes should still be vector-like by the analytic continuation of the charged and neutral Dirac propagators (116) and (120) from $p \neq 0$ to $p=\tilde{p}=0$. This argument is indeed correct in the phase diagram where $g_{1} \gg 1$ since we have a sensible strong-coupling limit, as we have shown in section 4. While, in the segment $\mathrm{A}\left(g_{2} \gg 1\right)$ that we speculate to be the scaling region of lattice chiral fermions, we are still lacking knowledge and evidence concerning the analyticity of the strong-coupling limit (95) and the spectrum (116, 120) around the point $p \simeq 0$. The problem that confronts us is how the three-fermion states 
that are composed of the normal modes of $\psi_{L}^{i}$ and $\psi_{R}$ disappear. If these threefermion states were resonant states, the vacuum would be unstable. The only loophole would appear if the propagators of interpolating fields (three-fermion states) vanished at $p=\tilde{p}=0$. But, this might describe massless ghost states with negative norm that couple to a gauge field, leading to an inconsistent theory [18]. We leave this discussion open in this paper 5 .

However, we would like to look at this point based on the point of view that is the essential idea presented in the original paper of Eichten and Preskill [2] to have chiral fermions in continuum limit. The question of whether the spectra of normal modes of $\psi_{L}^{i}(x)$ and $\psi_{R}(x)$ are massless and chiral is crucially related to the question of whether the normal modes of the three-fermion states $\left(\bar{\psi}_{R}(x)\right.$. $\left.\psi_{L}^{j}(x)\right) \psi_{R}(x)$ and $\left(\bar{\psi}_{L}^{i}(x) \cdot \psi_{R}(x)\right) \psi_{L}^{i}(x)$ have been composed in segment $A$. As have been qualitatively discussed in section 5 , the effective multifermion coupling (the 1PI four-point vertex function (72) ) is strongly momentum dependent and thresholds and wedges for different modes emerge in the phase diagram. This effective multifermion coupling for normal modes is very small in segment $A$, thus preventing the normal modes of the $\psi_{L}^{i}$ and $\psi_{R}$ from binding into threefermion states $\left(\bar{\psi}_{L}^{i} \cdot \psi_{R}\right) \psi_{L}^{i},\left(\bar{\psi}_{R} \cdot \psi_{L}^{i}\right) \psi_{R}$. We can reasonably speculate that there exists such a segment $A$ with finite $g_{2}$, where the scaling window of the continuum limit of chiral fermions would be opened up, although we cannot give a rigorous demonstration for the time being.

If what we expect is convincingly confirmed, the spectrum of the theory in this segment will be the following. The spectrum consists of 15 copies of $S U(2)$ QCD charged Dirac doublers $(p \neq 0)$ eq. (116) and 15 copies of $S U(2)$ neutral Dirac doublers $(p \neq 0)$ eq. (120). They are very massive and decoupled. Besides, the low-energy spectrum contain the two massless Weyl modes eqs. (20,21) for $g_{1}=0$ and $p=\tilde{p}$,

$$
S_{L}^{-1}(\tilde{p})^{i j}=i \gamma_{\mu} \tilde{p}^{\mu} \tilde{Z}_{2} \delta_{i j} P_{L} ; \quad S_{R}^{-1}(\tilde{p})=i \gamma_{\mu} \tilde{p}^{\mu} P_{R}
$$

which is in agreement with the $S U_{L}(2) \otimes U_{R}(1)$ symmetry. Namely, this normal mode of $\psi_{L}^{i}$ is self-scattering via the multifermion coupling $g_{2}$ (see Fig. 5) without pairing up with any other modes. The wave-function renormalization $\tilde{Z}_{2}$ can be considered as an interpolating constant of $Z_{2}(p)$ eq. (38) for $p=\tilde{p} \simeq 0$ and $g_{1}=0$. In addition, there is the $S U_{L}(2) \otimes U_{R}(1)$ covariant scalar $\mathcal{A}^{i}$ eq. (81). In order to see all possible interactions between these modes in this possible scaling region, we consider the one-particle irreducible vertex functions of these modes. In the light of the exact $S U_{L}(2) \otimes U_{R}(1)$ chiral symmetry and $\psi_{R}$-shift-symmetry, one can straightforwardly obtain non-vanishing vertex functions ( $d=$ dimensions) at physical momenta $(p=\tilde{p}, q=\tilde{q})$ : (i) $\mathcal{A}^{j} \mathcal{A}^{j \dagger} \mathcal{A}^{i} \mathcal{A}^{i \dagger}(d=4)$; (ii) $\bar{\psi}_{L}^{i} \psi_{L}^{i} \mathcal{A}^{j} \mathcal{A}^{j \dagger}$, $\bar{\Psi}_{c}^{i} \Psi_{c}^{i} \mathcal{A}^{j} \mathcal{A}^{j \dagger}$ and $\bar{\Psi}_{n} \Psi_{n} \mathcal{A}^{j} \mathcal{A}^{j \dagger}(d=5)$, as well as $d>5$ vertex functions. The

\footnotetext{
${ }^{5}$ I am grateful to N.B. Nielsen and Y. Shamir for discussions and sharing their wisdom on this point.
} 
vertex functions with dimensions $d>4$ vanish in the scaling region as $O\left(a^{d-4}\right)$ and we are left with the self-interacting vertex $\mathcal{A}^{j} \mathcal{A}^{j \dagger} \mathcal{A}^{i} \mathcal{A}^{i \dagger}$.

In this possible scaling region, the chiral continuum limit is very much like that of lattice QCD. We need to tune only one coupling $g_{1} \rightarrow 0$ in the neighbourhood of the segment A $g_{2}^{c, a}<g_{2}<g_{2}^{c, \infty}$. For $g_{1} \rightarrow 0$, the $\psi_{R}$ shift-symmetry is slightly violated, the normal modes of $\psi_{L}^{i}$ and $\psi_{R}$ would couple together to form the chiral symmetry breaking term $\Sigma^{i}(0) \bar{\psi}_{L}^{i} \psi_{R}$, which is a dimension-3 renormalized operator and thus irrelevant at the short distance. We desire this scaling region to be ultra-violet stable, in which the multifermion coupling $g_{1}$ turns out to be an effective renormalized dimension-4 operator [19].

\section{Some remarks}

The conclusion of the existence of the possible scaling region (90) for the continuum chiral theory is plausible and hard to be excluded. It is worthwhile to check this scenario in different approaches. Even though this scenario emerges, we are still left with several other problems to have a correct continuum chiral gauge theory when the global $S U_{L}(2)$ chiral symmetry is gauged. Their possible resolutions are mentioned and discussed in this section, and deserve to be studied in future work.

The question is whether this chiral continuum theory in the scaling region could be the correct chiral gauge theory, as the $S U(2)$ chiral gauge coupling $g$ is perturbatively turned on in the theory (1). One should expect a slight change of critical lines (points). We should be able to re-tune the multifermion couplings $\left(g_{1}, g_{2}\right)$ to compensate these perturbative changes, due the fact that the gauge interaction does not spoil the $\psi_{R}$ shift-symmetry and we can derive identities based on the Ward identity (15),

$$
\frac{\delta^{(2)} \Gamma}{\delta A_{\mu}^{\prime} \delta \bar{\psi}_{R}^{\prime}}=\frac{\delta^{(3)} \Gamma}{\delta A_{\mu}^{\prime} \delta \psi_{R}^{\prime} \delta \bar{\psi}_{R}^{\prime}}=\frac{\delta^{(3)} \Gamma}{\delta A_{\mu}^{\prime} \delta \Psi_{L}^{\prime n} \delta \bar{\psi}_{R}^{\prime}}=\cdots=0
$$

where $A_{\mu}^{\prime}$ is a "prime" gauge field. In this possible scaling regime, disregarding those uninteresting neutral modes, we have the charged modes including both the $S U(2)$ chiral-gauged, massless normal mode (121) of $\psi_{L}^{i}$ and the fifteen $S U(2)$ vectorial-gauged, massive doublers of the Dirac fermion $\Psi_{c}^{i}(116)$. The gauge field should not only chirally couple to the massless normal mode of the $\psi_{L}^{i}$ in the low-energy regime, but also vectorially couple to the massive doublers of Dirac fermion $\Psi_{c}^{i}$ in the high-energy regime. Thus, we expect the coupling vertex of the $S U_{L}(2)$-gauge field and the normal mode of the $\psi_{L}^{i}$ to be chiral at the continuum limit. We should be able to demonstrate this point on the basis of the Ward identities associated with the $S U(2)$ chiral gauge symmetry that is respected by the spectrum in the possible scaling regime. In fact, due to the reinstatement of the manifest $S U_{L}(2)$ chiral gauge symmetry and corresponding 
Ward identities of the undoubled spectrum in this possible scaling regime, we should then apply the Rome approach [20] (which is based on the conventional wisdom of quantum field theories) to perturbation theory in the small gauge coupling. It is expected that the Rome approach would work in the same way but all gauge-variant counterterms are prohibited; the gauge boson masses vanish to all orders of gauge coupling perturbation theory for $g_{1}=0$. We hope not to run into an inconsistent theory if the propagators of the three-fermion states which are composed of the normal modes of $\psi_{L}^{i}$ and $\psi_{R}$, positively vanish when $p \rightarrow 0$, i.e., the zero of these propagators at $p=0$ is a double zero.

Another important question remaining is how chiral gauge anomalies emerge, although in this short report the chiral gauge anomaly is cancelled by purposely choosing an appropriate fermion representation of the $S U_{L}(2)$ chiral gauge group. We know that in the doubled spectrum of naive lattice chiral gauge theory, the reason for the correct anomaly disappearing in the continuum limit is that the normal mode and doublers of Weyl fermions produce the same anomaly, so that these anomalies eliminate themselves [21]. As a consequence of decoupled doublers being given a chiral-invariant mass $\left(\sim O\left(\frac{1}{a}\right)\right)$, the surviving normal mode of the Weyl fermion (chiral-gauged, e.g., $U_{L}(1)$ ) should produce the correct anomaly in the continuum limit. Due to the fact that the action (II) possesses explicit $U_{L}(1)$ global symmetry, we also have the question of whether the conservation of left-handed fermion number would be violated by the correct anomaly [Z, 22] structure $\operatorname{tr} F \tilde{F}$ that is generated by the $S U(2)$ instanton in the continuum limit. We will discuses this crucial problem in a separate paper.

I thank G. Preparata, M. Creutz and H.B. Nielsen for many discussions. Thanks to R. Shrock and M. Testa for discussions on multifermion couplings, and P.G. Ratcliffe for reading this paper. The author gratefully acknowledges the support of K.C. Kong education of foundation, Hong Kong and the national foundation of science, China.

\section{Appendix I}

To obtain eq. (16), we need to consider

$$
\begin{aligned}
\left\langle\bar{\psi}_{L}^{i \alpha}(x) \psi_{R}^{\alpha}(x) \psi_{L}^{i \beta}(x)\right\rangle & \equiv-\frac{1}{Z} \frac{\delta}{\delta \eta_{L}^{i \alpha}(x)} \frac{\delta}{\delta \bar{\eta}_{R}^{\alpha}(x)} \frac{\delta}{\delta \bar{\eta}_{L}^{i \beta}(x)} Z \\
& =\frac{1}{Z} \frac{\delta}{\delta \eta_{L}^{i \alpha}(x)} \frac{\delta}{\delta \bar{\eta}_{L}^{i \beta}(x)}\left[Z \frac{1}{Z} \frac{\delta}{\delta \bar{\eta}_{R}^{\alpha}(x)} Z\right] \\
& =\frac{1}{Z} \frac{\delta}{\delta \eta_{L}^{i \alpha}(x)} \frac{\delta}{\delta \bar{\eta}_{L}^{i \beta}(x)}\left[Z(-) \psi_{R}^{\prime \alpha}(x)\right],
\end{aligned}
$$


where we utilize the relation (10), then

$$
\begin{aligned}
\frac{\delta}{\delta \psi_{R}^{\prime \alpha}(0)}\left\langle\bar{\psi}_{L}^{i \sigma}(x) \psi_{R}^{\sigma}(x) \psi_{L}^{i \beta}(x)\right\rangle & =-\frac{1}{Z} \frac{\delta}{\delta \eta_{L}^{i \alpha}(x)} \frac{\delta}{\delta \bar{\eta}_{L}^{i \beta}(x)}[Z \delta(x)], \\
& \equiv-\left\langle\bar{\psi}_{L}^{i \alpha}(x) \psi_{L}^{i \beta}(x)\right\rangle \delta(x) .
\end{aligned}
$$

Analogously, one can have,

$$
\frac{\delta}{\delta \psi_{R}^{\prime \alpha}(0)}\left\langle\Delta\left(\bar{\psi}_{L}^{i \sigma}(x) \Delta\left(\psi_{R}^{\sigma}(x)\right) \psi_{L}^{i \beta}(x)\right)\right\rangle=-\Delta\left(\left\langle\bar{\psi}_{L}^{i \alpha}(x) \psi_{L}^{i \beta}(x)\right\rangle \Delta \delta(x)\right)
$$

eqs. (124,125) lead to eq. (16).

$\left\langle\overline{\psi_{L}^{i \alpha}}(x) \psi_{L}^{i \beta}(y)\right\rangle_{\circ}$ is a spinor matrix that can be expanded on the basis of $I, \gamma_{\mu}, \sigma_{\mu \nu}, \gamma_{5} \gamma_{\mu}$ and $\gamma_{5}$, non-vanishing terms can be written as

$$
\begin{aligned}
\left\langle\bar{\psi}_{L}^{i \alpha}(x) \psi_{L}^{i \beta}(y)\right\rangle_{\circ} & =\left(P_{L}\right)^{\alpha \sigma}\left\langle\bar{\psi}^{i \sigma}(x) \psi^{i \theta}(y)\right\rangle_{\circ}\left(P_{R}\right)^{\theta \beta} \\
& =\left(P_{L}\right)^{\alpha \sigma} \int_{p} e^{i p(x-y)}\left(A\left(\bar{p}^{2}\right)\left(\gamma_{\mu}\right)^{\sigma \theta} \bar{p}_{\mu}+B\left(\bar{p}^{2}\right)\left(\gamma_{5} \gamma_{\mu}\right)^{\sigma \theta} \bar{p}_{\mu}\right)\left(P_{R}\right)^{\theta \beta},
\end{aligned}
$$

where $\int_{p}=\int_{-\pi}^{\pi} \frac{d^{4} p}{(2 \pi)^{4}}$ and $\bar{p}_{\mu}=\sin \left(p_{\mu}\right)$ is the lattice momentum. For $x=y$, one gets eq. (17),

$$
\left\langle\bar{\psi}_{L}^{i \alpha}(x) \psi_{L}^{i \beta}(x)\right\rangle_{\circ}=0, \quad \Delta\left\langle\bar{\psi}_{L}^{i \alpha}(x) \psi_{L}^{i \beta}(x)\right\rangle_{\circ}=0 .
$$

because of an odd function integral.

To obtain eq. (24), analogously to eqs. (123, 124, 125), we need to calculate

$$
\begin{aligned}
\left\langle\bar{\psi}_{L}^{i \sigma}(x) \psi_{R}^{\sigma}(x) \psi_{L}^{i \beta}(x)\right\rangle & =\frac{1}{Z} \frac{\delta}{\delta \eta_{L}^{i \sigma}(x)} \frac{\delta}{\delta \bar{\eta}_{R}^{\sigma}(x)}\left[Z \psi_{L}^{i \beta}(x)\right], \\
\frac{\delta}{\delta \psi_{L}^{\prime i \alpha}(0)}\left\langle\bar{\psi}_{L}^{i \sigma}(x) \psi_{R}^{\sigma}(x) \psi_{L}^{i \beta}(x)\right\rangle & =\left\langle\bar{\psi}_{L}^{i \sigma}(x) \psi_{R}^{\sigma}(x)\right\rangle \delta(x) \delta_{\alpha \beta},
\end{aligned}
$$

and

$$
\frac{\delta}{\delta \psi_{L}^{\prime i \alpha}(0)}\left\langle\Delta\left(\bar{\psi}_{L}^{i \sigma}(x) \Delta\left(\psi_{R}^{\sigma}(x)\right) \psi_{L}^{i \beta}(x)\right)\right\rangle=-\Delta\left(\left\langle\bar{\psi}_{L}^{i \sigma}(x) \psi_{R}^{\sigma}(x)\right\rangle \Delta \delta(x)\right) \delta_{\alpha \beta} .
$$

Armed with these equations, we obtain eq. (24) in section 3.

\section{Appendix II}

We re-insert the parametrization of (30) into the RHS of gap-equation (29), and equaling this to eq. (30) gives us two equations

$$
\begin{aligned}
\rho & =4 \tilde{g}_{1} \int_{q} \frac{\left(\tilde{g}_{2} w(q)+\rho\right)}{\sum_{\rho} \sin ^{2} q_{\rho}+\left(\rho+\tilde{g}_{2} w(q)\right)^{2}\left(v^{i} a\right)^{2}} \\
1 & =4 \int_{q} \frac{\left(\tilde{g}_{2} w(q)+\rho\right)}{\sum_{\rho} \sin ^{2} q_{\rho}+\left(\rho+\tilde{g}_{2} w(q)\right)^{2}\left(v^{i} a\right)^{2}} w(q) .
\end{aligned}
$$


With the definition $I_{n}$ (32) for $n=0,1,2$, we have equations

$$
\begin{aligned}
\rho & =\tilde{g}_{1} \tilde{g}_{2} I_{1}+\tilde{g}_{1} \rho I_{\circ} \\
1 & =4 \tilde{g}_{2} I_{2}+4 \rho I_{1},
\end{aligned}
$$

which lead to eq. (31).

The free propagator of $\psi_{L}^{i}$ is given as

$$
S_{\circ}^{i j}(p)=\delta_{i j} P_{L} \hat{p} P_{R}, \quad \hat{p}=\frac{\frac{i}{a} \sum_{\mu} \gamma^{\mu} \sin p_{\mu}}{\frac{1}{a^{2}} \sum_{\mu} \sin ^{2} p_{\mu}} a^{2} .
$$

The Feynman diagram (see Fig. 4) is given by,

$$
\begin{aligned}
\hat{\sigma}^{i j}(p)= & \delta^{i j} P_{R} \sigma(p) P_{L} \\
\sigma^{i j}(p)= & -a^{-2} \int_{k} \frac{\frac{i}{a} \sum_{\mu} \gamma^{\mu} \sin (p-k)_{\mu}}{\frac{1}{a^{2}} \sum_{\mu} \sin ^{2}(p-k)_{\mu}} \\
& \cdot \int_{q} \operatorname{tr}\left[P_{L} \frac{\frac{i}{a} \sum_{\mu} \gamma^{\mu} \sin \left(k-\frac{q}{2}\right)_{\mu}}{\frac{1}{a^{2}} \sum_{\mu} \sin ^{2}\left(k-\frac{q}{2}\right)_{\mu}} P_{R} \frac{\frac{i}{a} \sum_{\mu} \gamma^{\mu} \sin \left(k+\frac{q}{2}\right)_{\mu}}{\frac{1}{a^{2}} \sum_{\mu} \sin ^{2}\left(k+\frac{q}{2}\right)_{\mu}} P_{L}\right] \lambda \\
= & 2 a N_{f} \int_{k, q} \lambda \frac{i \sum_{\mu} \gamma^{\mu} \sin (p-k)_{\mu}}{\sum_{\mu} \sin ^{2}(p-k)_{\mu}} R(k, q),
\end{aligned}
$$

where $R(k, q)$ is eq. (38) and

$$
\lambda=\left(g_{1}+4 g_{2} w(p-k) w\left(k+\frac{q}{2}\right)\right)^{2}=\frac{1}{N_{f}^{2}} a^{-4}\left(\tilde{g}_{1}+4 \tilde{g}_{2} w(p-k) w\left(k+\frac{q}{2}\right)\right)^{2} .
$$

The wave-function renormalization $Z_{2}(p)$ of $\psi_{L}^{i}(x)$ in eq. (23) can be calculated by using the train approximation (see Fig. 5),

$$
\begin{aligned}
Z_{2}^{-1} S_{\circ}^{i j}(p) & =P_{L}(\hat{p}+\hat{p} \sigma \hat{p}+\hat{p} \sigma \hat{p} \sigma \hat{p}+\cdots) P_{R} \delta^{i j} \\
& =S_{\circ}^{i j}(p)\left(\frac{1}{1-\sigma \hat{p}}\right),
\end{aligned}
$$

and one gets

$$
Z_{2}=1-\sigma \hat{p}
$$

With eq. (133) one can get

$$
\sigma \hat{p}=-\frac{2}{N_{f}} \int_{k, q}\left(\tilde{g}_{1}+4 \tilde{g}_{2} w(p-k) w\left(k+\frac{q}{2}\right)\right)^{2} \frac{\sum_{\mu \nu} \gamma_{\mu} \gamma_{\nu} \sin (p-k)^{\mu} \sin p^{\nu}}{\sum_{\lambda \rho} \sin ^{2}(p-k)_{\lambda} \sin ^{2} p_{\rho}} R(k, q)
$$

By substituting eq. (137) into (136), one gets eq. (38).

\section{Appendix III}


We keep in mind that $\psi_{L}^{i}$ has two components and $\psi_{R}$ has one component (all times a factor of 2 for spin degeneracy). In the strong-coupling limit, the kinetic terms $S_{f}(x)$ :

$$
\begin{aligned}
& S_{f}(x)=S_{f}^{L}(x)+S_{f}^{R}(x) \\
& S_{f}^{L}(x)=\frac{1}{2 a g_{2}^{\frac{1}{2}}} \sum_{\mu} \bar{\psi}_{L}^{i}(x) \gamma_{\mu} \partial^{\mu} \psi_{L}^{i}(x) ; \quad S_{f}^{R}(x)=\frac{1}{2 a g_{2}^{\frac{1}{2}}} \sum_{\mu} \bar{\psi}_{R}(x) \gamma_{\mu} \partial^{\mu} \psi_{R}((x) 38)
\end{aligned}
$$

are dropped, the interacting action $S_{2}(x)$ (94) turns out to be bilinear in $\psi_{L}^{i}(x)$ fermion field at the same point " $x$ ". We first perform the integral of $\psi_{L}^{i}(x)(i$ fixed) at the point " $x$ " and obtain

$$
\begin{aligned}
D^{i}(x) & \equiv \Pi_{\alpha} \int\left[d \bar{\psi}_{L}^{i \alpha} d \psi_{L}^{i \alpha}\right] \exp \left(-\bar{\psi}_{L}^{i}(x) \cdot\left[\Delta \psi_{R}(x)\right]\left[\Delta \bar{\psi}_{R}(x)\right] \cdot \psi_{L}^{i}(x)\right) \\
& =\operatorname{det}_{s}\left(\begin{array}{cc}
\Delta \bar{\psi}_{R}^{1}(x) \Delta \psi_{R}^{1}(x) & \Delta \bar{\psi}_{R}^{1}(x) \Delta \psi_{R}^{2}(x) \\
\Delta \bar{\psi}_{R}^{2}(x) \Delta \psi_{R}^{1}(x) & \Delta \bar{\psi}_{R}^{2}(x) \Delta \psi_{R}^{2}(x)
\end{array}\right) \\
& =2 \Pi_{\alpha} \Delta \psi_{R}^{\alpha}(x) \Delta \bar{\psi}_{R}^{\alpha}(x),
\end{aligned}
$$

where the determent $\operatorname{det}_{s}$ is taken over spinor space. Then, the partition function of the one-site theory is given by the integral that is bilinear in fermionic variable $\psi_{R}^{\alpha}(x)$,

$$
\begin{aligned}
Z^{i}(x) & =\Pi_{\alpha} \int\left[d \bar{\psi}_{R}^{\alpha}(x) d \psi_{R}^{\alpha}(x)\right] D^{i}(x) \\
& =\Pi_{\alpha}\left(\int\left[d \bar{\psi}_{R}^{\alpha}(x) d \psi_{R}^{\alpha}(x)\right] 2 \Delta \psi_{R}^{\alpha}(x) \Delta \bar{\psi}_{R}^{\alpha}(x)\right) \\
& =2^{2} \operatorname{det}_{s}\left(\Delta^{2}(x)\right)=2^{2}\left(\Delta^{2}(x)\right)^{2} .
\end{aligned}
$$

The total partition function in the strong-coupling limit is then obtained,

$$
\begin{aligned}
Z & =\Pi_{i, x}\left(\Pi_{\alpha} \int\left[d \bar{\psi}_{R}^{\alpha}(x) d \psi_{R}^{\alpha}(x)\right] 2 \Delta \psi_{R}^{\alpha}(x) \Delta \bar{\psi}_{R}^{\alpha}(x)\right) \\
& =2^{4 N}\left(\operatorname{det}_{x} \Delta^{2}(x)\right)^{4}
\end{aligned}
$$

where the determent $\operatorname{det}_{x}$ is taken only over the lattice space-time and $N$ is the number of lattice sites.

In order to obtain the recursion relations (57,58,59), (105, 106, 107) and (77) satisfied by two-point functions, we consider an integral of one field $\psi_{L}^{3}(x)$ at the point " $x$ " defined as

$$
P_{1}^{j \sigma}(x) \equiv \frac{1}{Z^{j}(x)} \int_{x}^{R} \int_{x j}^{L} \bar{\psi}_{L}^{j \sigma}(x) e^{-S_{f}(x)-S_{2}^{j}(x)},
$$

where the measure of the fermion fields $\psi_{L}^{j}(x)$ (the $S U_{L}(2)$-index $j$ is fixed) and $\psi_{R}(x)$ at the point " $x$ " is given as

$$
\int_{x}^{R} \int_{x j}^{L} \equiv \Pi_{\alpha} \int\left[d \bar{\psi}_{R}^{\alpha}(x) d \psi_{R}^{\alpha}(x)\right]\left[d \bar{\psi}_{L}^{j \alpha}(x) d \psi_{L}^{j \alpha}(x)\right] .
$$


To have a non-vanishing integral of $\psi_{L}^{j}(x)$ at the point " $x$ ", we need a $\psi_{L}^{j}(x)$ field in the expansion of $e^{-S_{f}^{L}(x)}$, and obtain

$$
P_{1}^{j \sigma}(x)=\frac{1}{Z^{j}(x)}\left(\frac{1}{2 a g_{2}^{\frac{1}{2}}}\right) \sum_{\mu}^{\dagger}\left[\bar{\psi}_{L}^{j}(x+\mu) \gamma_{\mu}\right]^{\gamma} \int_{x}^{R} \int_{x j}^{L} \psi_{L}^{j \gamma}(x) \bar{\psi}_{L}^{j \sigma}(x) e^{-S_{f}^{R}(x)-S_{2}^{j}(x)}
$$

where

$$
\sum_{\mu}^{\dagger}\left[\bar{\psi}_{L}^{j}(x+\mu) \gamma_{\mu}\right]^{\gamma}=\left[\bar{\psi}_{L}^{j}(x+\mu) \gamma_{\mu}\right]^{\gamma}-\left[\bar{\psi}_{L}^{j}(x-\mu) \gamma_{\mu}\right]^{\gamma} .
$$

Using eq. (139), we can first perform the integral over $\psi_{L}^{j}(x)$ in eq. (144) that is bilinear in terms of $\psi_{L}^{j}(x)$,

$$
\begin{aligned}
\int_{x j}^{L} \psi_{L}^{j \gamma}(x) \bar{\psi}_{L}^{j \sigma}(x) e^{-S_{2}^{j}(x)} & =\left(\frac{D^{j}(x)}{\Delta \psi_{R}^{\gamma}(x) \Delta \bar{\psi}_{R}^{\sigma}(x)}\right) \\
\left(\frac{D^{j}(x)}{\Delta \psi_{R}^{\gamma}(x) \Delta \bar{\psi}_{R}^{\sigma}(x)}\right) & =-\delta_{\gamma 1} \delta_{\sigma 1} \Delta \psi_{R}^{2}(x) \Delta \bar{\psi}_{R}^{2}(x)-\delta_{\gamma 2} \delta_{\sigma 2} \Delta \psi_{R}^{1}(x) \Delta \bar{\psi}_{R}^{1}(x) \\
& +\delta_{\gamma 1} \delta_{\sigma 2} \Delta \psi_{R}^{2}(x) \Delta \bar{\psi}_{R}^{1}(x)+\delta_{\gamma 2} \delta_{\sigma 1} \Delta \psi_{R}^{1}(x) \Delta \bar{\psi}_{R}^{2}(x)
\end{aligned}
$$

As a result, we have

$$
P_{1}^{j \sigma}(x)=\left(\frac{1}{2 a g_{2}^{\frac{1}{2}}}\right) \sum_{\mu}^{\dagger}\left[\bar{\psi}_{L}^{j}(x+\mu) \gamma_{\mu}\right]^{\gamma} \frac{1}{Z^{j}(x)} \int_{x}^{R} e^{-S_{f}^{R}(x)}\left(\frac{D^{j}(x)}{\Delta \psi_{R}^{\gamma}(x) \Delta \bar{\psi}_{R}^{\sigma}(x)}\right)
$$

The remaining integral (147) of $\psi_{R}(x)$ at the " $x$ " point is bilinear in terms of fermionic variable $\psi_{R}^{\alpha}(x)$, we need to have $\psi_{R}(x)$ and $\bar{\psi}_{R}(x)$ fields in expansion of $e^{-S_{f}^{R}(x)}$, for the lowest order of $O\left(\frac{1}{g_{2}}\right)$, we obtain

$$
P_{1}^{j \sigma}(x)=\left(\frac{1}{2 a g_{2}^{\frac{1}{2}}}\right)^{3} \sum_{\mu}^{\dagger}\left[\bar{\psi}_{L}^{j}(x+\mu) \gamma_{\mu}\right]^{\gamma}\left[\gamma_{\mu} \psi_{R}(x+\mu)\right]^{\alpha}\left[\bar{\psi}_{R}(x+\mu) \gamma_{\mu}\right]^{\beta} \Theta_{\beta \alpha \gamma \sigma}^{j}(x),
$$

where

$$
\Theta_{\beta \alpha \gamma \sigma}^{j}(x)=\frac{1}{Z^{j}(x)} \Pi_{\lambda} \int\left[d \bar{\psi}_{R}^{\lambda}(x) d \psi_{R}^{\lambda}(x)\right] \psi_{R}^{\beta}(x) \bar{\psi}_{R}^{\alpha}(x)\left(\frac{D^{j}(x)}{\Delta \psi_{R}^{\gamma}(x) \Delta \bar{\psi}_{R}^{\sigma}(x)}\right) .
$$

Using eqs. (140,146) and the following relation

$$
\begin{aligned}
& \frac{1}{Z^{j}(x)} \Pi_{\lambda} \int\left[d \bar{\psi}_{R}^{\lambda}(x) d \psi_{R}^{\lambda}(x)\right]\left[\Delta \psi_{R}^{\gamma}(x) \Delta \bar{\psi}_{R}^{\sigma}(x)\right] \psi_{R}^{\beta}(x) \bar{\psi}_{R}^{\alpha}(x) \\
= & \frac{1}{Z^{j}(x)} \frac{\delta_{\beta \sigma} \delta_{\gamma \alpha}}{\Delta^{2}(x)} 2^{2} \operatorname{det}_{s}\left(\Delta^{2}(x)\right)=\frac{\delta_{\beta \sigma} \delta_{\gamma \alpha}}{\Delta^{2}(x)}
\end{aligned}
$$


we obtain

$$
\begin{aligned}
\Theta_{\beta \alpha \gamma \sigma}^{j} & =\frac{\delta_{\beta \sigma} \delta_{\gamma \alpha}}{\Delta^{2}(x)} \\
P_{1}^{j \sigma}(x) & =\frac{1}{\Delta^{2}(x)}\left(\frac{1}{2 a g_{2}^{\frac{1}{2}}}\right)^{3} \sum_{\mu}^{\dagger}\left[\bar{\psi}_{L}^{j}(x+\mu) \cdot \psi_{R}(x+\mu)\right]\left[\bar{\psi}_{R}(x+\mu) \gamma_{\mu}\right]^{\sigma} .
\end{aligned}
$$

In eq. (152) the reason for the three fields $\psi_{R}, \bar{\psi}_{R}$ and $\psi_{L}^{i}$ being at the same point " $x+\mu$ " is due to the lowest non-trivial approximation.

We define the integral of three fields $P_{3}^{j \sigma}(x)$ at the site " $x$ "

$$
P_{3}^{j \sigma}(x) \equiv \frac{1}{Z^{j}(x)} \int_{x}^{R} \int_{x j}^{L} \bar{\psi}_{L}^{j \alpha}(x) \psi_{R}^{\alpha}(x) \bar{\psi}_{R}^{\sigma}(x) e^{-S_{f}(x)-S_{2}^{j}(x)} .
$$

Analogously to the reason for eqs. (147,149) to get non-trivial results, we only need a $\psi_{L}^{j}(x)$ field in the expansion of $e^{-S_{f}^{L}(x)}$, and considering eqs. (146, 151) we obtain,

$$
\begin{aligned}
P_{3}^{j \sigma}(x) & =\frac{1}{Z^{j}(x)}\left(\frac{1}{2 a g_{2}^{\frac{1}{2}}}\right) \sum_{\mu}^{\dagger}\left[\bar{\psi}_{L}^{j}(x+\mu) \gamma_{\mu}\right]^{\gamma} \int_{x}^{R} \int_{x j}^{L} \psi_{L}^{j \gamma}(x) \bar{\psi}_{L}^{j \alpha}(x) \psi_{R}^{\alpha}(x) \bar{\psi}_{R}^{\sigma}(x) e^{-S_{2}^{j}(x)} \\
& =\left(\frac{1}{2 a g_{2}^{\frac{1}{2}}}\right) \sum_{\mu}^{\dagger}\left[\bar{\psi}_{L}^{j}(x+\mu) \gamma_{\mu}\right]^{\gamma} \Theta_{\beta \sigma \gamma \beta}^{j}(x) \\
& =\frac{1}{\Delta^{2}(x)}\left(\frac{1}{2 a g_{2}^{\frac{1}{2}}}\right) \sum_{\mu}^{\dagger}\left[\bar{\psi}_{L}^{j}(x+\mu) \gamma_{\mu}\right]^{\sigma}
\end{aligned}
$$

We turn to consider the integral of the four fermion fields at site " $x$ ",

$$
\begin{aligned}
P_{4}^{i j, \theta \sigma}(x) & \equiv \frac{1}{Z^{j}(x)} \int_{x}^{R} \int_{x j}^{L} \psi_{L}^{\theta i}(0) \bar{\psi}_{L}^{j \alpha}(x) \psi_{R}^{\alpha}(x) \bar{\psi}_{R}^{\sigma}(x) e^{-S_{f}(x)-S_{2}^{j}(x)} \\
& \simeq \frac{1}{Z^{j}(x)} \int_{x}^{R} \int_{x j}^{L} \psi_{L}^{i \theta}(0) \bar{\psi}_{L}^{j \alpha}(x) \psi_{R}^{\alpha}(x) \bar{\psi}_{R}^{\sigma}(x) e^{-S_{2}^{j}(x)} \\
& =\delta(x) \delta_{i j} \Theta_{\alpha \sigma \theta \alpha}(x) \\
& =\frac{\delta_{\theta \sigma} \delta(x) \delta_{i j}}{\Delta^{2}(x)}
\end{aligned}
$$

We compute the following integral $P_{2}^{j}(\mathrm{x})$ of two fields $\bar{\psi}_{L}^{j \alpha}(x) \psi_{R}^{\alpha}(x)$ at " $x^{\prime}$,

$$
P_{2}^{j}(x) \equiv \frac{1}{Z^{j}(x)} \int_{x}^{R} \int_{x j}^{L} \bar{\psi}_{L}^{j \alpha}(x) \psi_{R}^{\alpha}(x) e^{-S_{f}(x)-S_{2}^{j}(x)} .
$$

To have a non-trivial result, we need $\psi_{L}^{j}(x)$ and $\psi_{R}(x)$ fields in the expansion of $e^{-S_{f}(x)}$, and we obtain

$$
P_{2}^{j}(x)=\frac{1}{Z^{j}(x)}\left(\frac{1}{2 a g_{2}^{\frac{1}{2}}}\right) \sum_{\mu}^{\dagger}\left[\bar{\psi}_{L}^{i}(x+\mu) \gamma_{\mu}\right]^{\gamma} \int_{x}^{R} \int_{x j}^{L} \psi_{L}^{j \gamma}(x) \bar{\psi}_{L}^{j \alpha}(x) \psi_{R}^{\alpha}(x) e^{-S_{f}^{R}(x)-S_{2}^{j}(x)}
$$




$$
\begin{aligned}
& =\left(\frac{1}{2 a g_{2}^{\frac{1}{2}}}\right)^{2} \sum_{ \pm \mu}\left[\bar{\psi}_{L}^{j}(x+\mu) \gamma_{\mu}\right]^{\gamma}\left[\gamma_{\mu} \psi_{R}(x+\mu)\right]^{\sigma} \Theta_{\alpha \sigma \gamma \alpha}(x) \\
& =\frac{1}{\Delta^{2}(x)}\left(\frac{1}{2 a g_{2}^{\frac{1}{2}}}\right)^{2} \sum_{ \pm \mu}\left[\bar{\psi}_{L}^{j}(x+\mu) \psi_{R}(x+\mu)\right]
\end{aligned}
$$

Armed with these equations (152,154,155, 157), one can get the recursion relations satisfied by two-point functions in the main text of the article.

\section{References}

[1] H.B. Nielsen and M. Ninomiya, Nucl. Phys. B185 (1981) 20, ibid. B193 (1981) 173, Phys. Lett. B105 (1981) 219.

[2] E. Eichten and J. Preskill, Nucl. Phys. B268 (1986) 179.

[3] M.F.L. Golterman, D.N. Petcher and E. Rivas, Nucl. Phys. B395 (1993) 597.

[4] J. Smit, Acta Physica Polonica B17 (1986) 531;

P.D.V. Swift, Phys. Lett. B145 (1984) 256.

[5] D.N. Petcher, Nucl. Phys.(Proc. Suppl.) B30 (1993) 52, references there in.

[6] G. Preparata and S.-S. Xue, Phys. Lett. B264 (1991) 35; Nucl. Phys. B26 (Proc. Suppl.) (1992) 501; Nucl. Phys. B30 (Proc. Suppl.) (1993) 647.

[7] A. Hasenfratz, P. Hasenfratz, K. Jansen, J. Kuti and Yue Shen, Nucl. Phys. B365 (1991) 79.

[8] M.F.L. Golterman, D.N. Petcher and J. Smit, Nucl. Phys. B370 (1992) 51.

[9] Y. Shamir, Phys. Rev. Lett. 71 (1993) 2691; Nucl. Phys.(Proc. Suppl.) B34 (1994) 590; hep-lat/9307002; Nucl. Phys.(Proc. Suppl.) B47 (1996) 212.

[10] H.B. Nielsen and M. Ninomiya, Int. J. of Mod. Phys. A6 (1991) 2913; H.B. Nielsen and S.E. Rugh, Nucl. Phys. 29B (Proc. Suppl.) (1992) 200.

[11] M. Creutz, Nucl. Phys.(Proc. Suppl.) B42 (1995) 56, Phys. Rev. D52 (1995) 2951.

[12] R. Narayanan and H. Neuberger, Nucl. Phys. B443 (1995) 305, references there in;

G. 't Hooft, Phys. Lett. B349 (1995) 491;

P. Hernández and R. Sundrum, Nucl. Phys. B455 (1995) 287. 
[13] M.F.L. Golterman, D.N. Petcher, Phys. Lett. B225 (1989) 159.

[14] Y. Nambu and G. Jona-Lasinio, Phys. Rev. 122 (1961) 345.

[15] G. Preparata and S.-S. Xue, Phys. Lett. B335 (1994) 192; ibid B377 (1996) 124.

[16] K. Wilson, in New phenomena in subnuclear physics (Erice, 1975) ed. A. Zichichi (Plenum, New York, 1977).

[17] The private communication with Y. Shamir.

[18] M. campostrini, G. Curci and A. Pelissetto, Phys. Lett. B193 (1987) 279; G.T. Bodwin and E.V. Kovacs, Phys. Lett. B193 (1987) 283;

A. Pelissetto, Ann. Phys. 182 (1988) 177.

[19] W.A. Bardeen, C.T. Hill and S. Love, Nucl. Phys. B273 (1986) 649. ibid B323 (1989) 493,

W.A.Bardeen, S. T. Love and V.A. Miransky, Phys. Rev. D42 (1990) 3514.

[20] A. Borrelli, L. Maiani, G.C. Rossi, R. Sisto and M. Testa, Nucl. Phys. B333 (1990) 335; Phys. Lett. B221 (1989) 360;

L. Maiani, G.C. Rossi, and M. Testa, Phys. Lett. B261 (1991) 479; ibid B292 (1992) 397;

L. Maiani, Nucl. Phys. (Proc.Suppl.) B29 (1992) 33.

[21] L. H. Karsten and J. Smit, Nucl. Phys. B144 (1978) 536.

[22] T. Banks, Phys. Lett. B272 (1991) 75; T. Bank and A. Dabholkar, Nucl. Phys. 29B (Proc. Suppl.) (1992) 46.

\section{Figure Captions}

Figure 1: The phase diagram for the theory (11) in the $g_{1}-g_{2}$ plane.

Figure 2: The effective four-point interacting vertex.

Figure 3: The NJL gap-equation for the self-energy function $\Sigma(p)$ in the limit of $N_{f} \rightarrow \infty$.

Figure 4: The Feynman diagram of the leading contribution to the wavefunction renormalization $Z_{2}(p)$ of $\psi_{L}^{i}$.

Figure 5: The train approximation to the wave-function renormalization $Z_{2}(p)$ of $\psi_{L}^{i}$. 